\title{
Infrared degrees of freedom of Yang-Mills theory in the Schrödinger representation
}

\author{
Hilmar Forkel \\ IFT, Universidade Estadual Paulista, Rua Pamplona, 145, 01405-900 São Paulo, SP, Brazil \\ ECT*, Strada delle Tabarelle 286, I-38050 Villazzano (Trento), Italy \\ Institut für Theoretische Physik, Universität Heidelberg, D-69120 Heidelberg, Germany
}

(Received 17 December 2005; published 1 May 2006)

\begin{abstract}
We set up a new calculational framework for the Yang-Mills vacuum transition amplitude in the Schrödinger representation. After integrating out hard-mode contributions perturbatively and performing a gauge-invariant gradient expansion of the ensuing soft-mode action, a manageable saddle-point expansion for the vacuum overlap can be formulated. In combination with the squeezed approximation to the vacuum wave functional this allows for an essentially analytical treatment of physical amplitudes. Moreover, it leads to the identification of dominant and gauge-invariant classes of gauge field orbits which play the role of gluonic infrared (IR) degrees of freedom. The latter emerge as a diverse set of saddle-point solutions and are represented by unitary matrix fields. We discuss their scale stability, the associated virial theorem and other general properties including topological quantum numbers and action bounds. We then find important saddle-point solutions (most of them solitons) explicitly and examine their physical impact. While some are related to tunneling solutions of the classical Yang-Mills equation, i.e. to instantons and merons, others appear to play unprecedented roles. A remarkable new class of IR degrees of freedom consists of Faddeev-Niemi type link and knot solutions, potentially related to glueballs.
\end{abstract}

DOI: 10.1103/PhysRevD.73.105002

PACS numbers: 11.15.- q, 11.15.Tk, 12.38.Lg

\section{INTRODUCTION}

The strong couplings among soft QCD gluons manifest themselves in a variety of complex long-distance phenomena which are thoroughly entwined with the vacuum state. Their diversity is well documented by prominent examples such as quark confinement, spontaneous chiral symmetry breaking, vacuum tunneling, the ensuing $\theta$ structure and gluon condensates. Nevertheless, one commonly expects the essence of the underlying dynamics to involve just a few types of soft gluonic modes.

The quest for these long-wavelength excitations began shortly after the inception of quantum chromodynamics (QCD) and has inspired the development of various kinds of vacuum models, based e.g. on glueball condensation [1], Gaussian stochastic processes [2], gluonic domains [3,4] as well as instanton [5], vortex [6], and meron [7] ensembles. Over the last decade, lattice simulations increasingly assisted in the search for predominant infrared (IR) gluon fields, mostly by means of numerical smoothing [8] and gauge-fixing [9] techniques. These simulations are beginning to generate quantitative insights into the role of instantons and their size distribution [10] as well as into the classic confinement scenarios based on (gaugeprojected Abelian) monopole $[9,11]$ or center vortex [6] condensation.

Nevertheless, at present no mechanism involving soft vacuum gluons can be uniquely or systematically related to QCD, and many crucial questions regarding the underlying fields, their stability, gauge-independent physical interpretation, mutual interactions, relations to other vacuum fields etc. remain unanswered. Analytical progress in this realm is hampered by the inevitable gauge dependence of the classical gluon-field configurations on which most of the existing proposals are based. Similarly, approaches which reformulate the Yang-Mills dynamics in terms of gaugeinvariant loop variables [12] or resolve the gauge constraints explicitly (e.g. in Coulomb gauge [13]), are technically too involved for most analytical applications.

In the present paper, we bypass these intricacies by developing an approach in which not the contributions of single gauge fields but rather those of gauge-invariant classes are treated jointly. These classes gather universal contributions from dominant gauge field orbits to lowenergy amplitudes, i.e. they play the role of collective gluonic IR degrees of freedom. More explicitly, they are represented by unitary matrix fields which emerge as saddle points of a generalized nonlinear $\sigma$-model action, encode a common relative gauge orientation of the underlying gluons and provide the principal input for a systematic expansion of soft amplitudes.

Manifest gauge invariance is maintained throughout all calculations by working in the Hamiltonian formulation of Yang-Mills theory, adopting the "coordinate" Schrödinger representation and making use of explicit gauge projection operators. The Schrödinger picture is chosen mainly because it restricts gauge transformations to a fixed reference time, thereby effectively decoupling them from the dynamical time evolution, and because it often exhibits the impact of topological properties in a particularly transparent way (even without recourse to the semiclassical approximation) [14]. In the present paper we will focus on gluonic effects and work in pure Yang-Mills theory without quarks.

Although our approach preserves explicitly traceable links between the soft collective fields and the underlying gauge fields, the resulting IR dynamics turns out to be 
reasonably transparent and to allow for an essentially analytical treatment of the saddle points. In contrast to effective theories (e.g. for Polyakov loops) which just share symmetries with their fundamental dynamics while all coupling parameters have to be fitted to experimental or lattice data, moreover, our soft-mode Lagrangian can be uniquely derived from the underlying vacuum wave functional.

The development of the saddle-point expansion framework will be split into two parts. The present paper contains the first one in which we formulate our approach, derive important saddle-point solution families explicitly and discuss their main properties and physical interpretations. The evaluation of contributions from specific IR degrees of freedom to soft Yang-Mills amplitudes, such as gluon condensates and the topological susceptibility, will appear separately [15]. This division suggests itself not only because the relevant saddle points have to be found before applications become feasible, but more importantly because the IR degrees of freedom are of substantial interest in their own right. In particular, their analysis and interpretation generates new insights into how soft vacuum gluons organize themselves into "collective variables." Under the related results are a virialtheorem based mechanism by which the extended IR fields stabilize their size, a new physical interpretation for Faddeev-Niemi theory and a gauge-invariant representation of instanton and meron effects together with the associated quantum fluctuations.

The paper is organized as follows: in Sec. II we recapitulate the definition of the vacuum overlap amplitude in the Schrödinger picture. We then implement a gaugeprojected vacuum wave functional on the basis of the Gaussian approximation and rewrite the overlap in terms of a bare action which had previously been used in a variational context. In Sec. III, we take advantage of known one-loop results to integrate out the hard-mode contributions to the bare action perturbatively. By means of a controlled derivative expansion, the renormalized softmode action density is then transformed into a local Lagrangian which greatly simplifies the further analysis. In Sec. IV we build on these results by setting up the IRsensitive saddle-point expansion for the functional integral over the soft modes and by deriving the saddle-point equations whose nontrivial solutions represent the gluonic IR degrees of freedom.

Important generic properties of these IR variables are established in Sec. V, including their scale stability due to a virial theorem, three topological quantum numbers and a lower bound of Bogomol'nyi type on their action. In Sec. VI, several classes of the more symmetric and most important saddle-point solutions are found explicitly. They comprise topological soliton solutions of hedgehog type, which are related to classical solutions of the Yang-Mills equation, and solutions which carry other topological in- formation and seem to have no obvious Yang-Mills counterparts. One of the most intriguing solution classes consists of solitonic links and knots. Those emerge from a generalization of Faddeev-Niemi theory which turns out to be embedded in our soft-mode Lagrangian. In Sec. VII we classify all hedgehog soliton solutions, find their most important representatives explicitly, and establish the role of the regular solutions as mainly summarizing contributions from instanton and meron gauge orbits. In Sec. VIII, finally, we collect our principal results, comment on evaluating contributions from gluonic IR degrees of freedom to relevant amplitudes, and suggest a few additional directions for future work.

\section{VACUUM OVERLAP AMPLITUDE}

The vacuum overlap amplitude of $\mathrm{SU}(N)$ Yang-Mills theory (without matter fields) in the Schrödinger coordinate representation [14] reads

$$
Z^{\prime}:=\left\langle 0, t_{+} \mid 0, t_{-}\right\rangle=\int D \vec{A} \Psi_{0}^{*}\left[\vec{A}, t_{+}\right] \Psi_{0}\left[\vec{A}, t_{-}\right],
$$

where $\Psi_{0}$, the vacuum wave functional (VWF), depends on half of the canonical variables, i.e. on the static gauge fields $\vec{A}(\vec{x})$. The gauge invariance of $\Psi_{0}$, like that of any other physical state and associated wave functional, is dictated by Gauss's law. This crucial constraint can be imposed on any given functional by projection onto its gauge-singlet component, i.e. by integration over the (compact) gauge group [16]. Starting from an approximate and therefore generally gauge-dependent wave functional $\psi_{0}$, this produces the associated VWF

$$
\Psi_{0}[\vec{A}]=\sum_{Q} e^{i Q \theta} \int D \mu\left(U^{(Q)}\right) \psi_{0}\left[\vec{A}^{U^{(Q)}}\right]=: \int D U \psi_{0}\left[\vec{A}^{U}\right]
$$

( $d \mu$ is the invariant Haar measure of the gauge group, $Q$ is the homotopy degree of the group element $U^{(Q)}$, and $\theta$ the vacuum angle) which aquires the obligatory $\theta$ phase under topologically nontrivial gauge transformations and renders Gauss's law manifest. The vacuum energy has been set to zero.

After interchanging the order of integrations over gauge fields and gauge group in Eq. (1), a gauge group volume can be factored out of $Z^{\prime}$, i.e.

$$
\begin{aligned}
Z^{\prime} & =\int D U_{+} \int D U_{-} \int D \vec{A} \psi_{0}^{*}\left[\vec{A}^{U_{+}}\right] \psi_{0}\left[\vec{A}^{U_{-}}\right] \\
& =: Z \int D U_{-},
\end{aligned}
$$

since the $\vec{A}$-integral is gauge invariant. (The group volume $Z^{\prime} / Z$ is left over when the two un-normalized gauge projectors in the matrix element $Z^{\prime}$ multiply into one.) The integrand of the remaining group integration is suitably rewritten as a Boltzmann factor, i.e. 


$$
Z=\int D U \exp \left(-\Gamma_{b}[U]\right)
$$

and defines the 3-dimensional Euclidean bare action $\Gamma_{b}$ as a functional of the "relative" gauge orientation $U \equiv$ $U_{-}^{-1} U_{+}$only. Owing to the gauge invariance of the gluon-field measure, $\Gamma_{b}$ is by itself gauge invariant and takes the explicit form

$$
\Gamma_{b}[U]=-\ln \int D \vec{A} \psi_{0}^{*}\left[\vec{A}^{U}\right] \psi_{0}[\vec{A}] .
$$

This action describes dynamical correlations which originate from the gauge projection in Eq. (2), i.e. $\Gamma_{b}$ would become $U$ independent if $\psi_{0}$ were gauge invariant by itself. More specifically, $\Gamma_{b}[U]$ gathers all those contributions to $Z$ whose approximate vacua $\psi_{0}$ at $t= \pm \infty$ differ by the relative gauge orientation $U$. Hence the variable $U$ represents contributions of a specifically weighted ensemble of all gluon-field orbits to the vacuum overlap and is gauge invariant by construction.

To proceed in an analytically tractable fashion, we now adopt the standard Gaussian ansatz

$\psi_{0}^{(G)}[\vec{A}]=\exp \left[-\frac{1}{2} \int d^{3} x \int d^{3} y A_{i}^{a}(\vec{x}) G^{-1 a b}(\vec{x}-\vec{y}) A_{i}^{b}(\vec{y})\right]$

for the unprojected $\mathrm{VWF}^{1}$. As expected from a ground state wave functional, $\psi_{0}^{(G)}$ has no nodes. Besides allowing integrals over $\vec{A}$ to be evaluated exactly, there are several physical reasons for choosing the Gaussian functional (6). To start with, it describes a "squeezed" state, i.e. an oscillator-type extension of the unstable coherent gluon states [19] and thus the simplest natural candidate for the vacuum functional. In fact, Eq. (6) turns into the exact ground state of $\mathrm{U}(1)$ gauge theory (up to color factors) if the "covariance" $G^{-1}$ is taken to be the inverse of the massless static vector propagator.

Several additional properties indicate that a Gaussian VWF with the appropriate covariance captures crucial features of the Yang-Mills dynamics as well. First of all, a suitable choice for $G^{-1}$ (see below) renders the wave functional (6) exact at high momenta and hence incorporates asymptotic freedom. Moreover, it is known from variational analyses that Gaussian VWFs generate a dynamical mass gap [20,21] and, with a suitable prefactor in Coulomb gauge, an approximately linearly rising confinement potential [22]. (Mass generation and other features of $2+1$ dimensional compact photodynamics are also reproduced [23].) Additional insight into and support for the

\footnotetext{
${ }^{1}$ For our exploratory purposes we do not retain a possible longitudinal contribution to $G^{-1}$ which was discussed in Ref. [17]. (A certain gauge-invariant extension of the exponent in Eq. (6) approximates the ground state of Yang-Mills theory in $2+1$ dimensions [18].)
}

Gaussian approximation will be supplied by our results below. Nevertheless, the full extent to which the Gaussian ansatz can approximate the VWFs of strongly coupled Yang-Mills theories, and similarly its nonperturbative limitations, remain at present unknown. In particular, it has not been shown that the functional (6), after gauge projection according to Eq. (2), generates the crucial area law for large, spacelike Wilson loops in the fundamental representation (although heuristic arguments to this end have been given in Ref. [24]) and how the behavior of the adjoint Wilson loop can be accomodated.

After specializing the bare action (5) to the Gaussian VWF, the integral over the gluon fields can readily be carried out. The result takes the form of a 3-dimensional, bilocal nonlinear sigma model [24],

$$
\Gamma_{b}[U]=\frac{1}{2 g_{b}^{2}} \int d^{3} x \int d^{3} y L_{i}^{a}(\vec{x}) D^{a b}(\vec{x}-\vec{y}) L_{i}^{b}(\vec{y}) .
$$

(Above we have omitted a term of higher order in the small bare coupling $g_{b}$.) The $U$-dependence enters $\Gamma_{b}$ both via the one-forms

$$
L_{i}(\vec{x})=U^{\dagger}(\vec{x}) \partial_{i} U(\vec{x})=: L_{i}^{a}(\vec{x}) \frac{\lambda^{a}}{2 i},
$$

i.e. the Lie-algebra valued, left-invariant Cartan-Maurer "currents" (with real components $L_{i}^{a}$ ), and through higher-order corrections to the bilocal operator

$$
D^{a b}=\left[\left(G+G^{U}\right)^{-1}\right]^{a b} \simeq \frac{1}{2} G^{-1} \delta^{a b}+\ldots,
$$

where $\quad G^{U}=G^{a b}(\vec{x}-\vec{y}) U^{\dagger}(\vec{x}) T^{a} U(\vec{x}) \otimes U(\vec{y}) T^{b} U^{\dagger}(\vec{y})$, $T^{a}=\lambda^{a} / 2$, and $G^{-1 a b}=G^{-1} \delta^{a b}$. The above reformulation of the Yang-Mills vacuum overlap on the basis of a gauge-invariant Gaussian VWF was employed in Ref. [24] as the starting point for a variational approach ${ }^{2}$. Alternatively, it can be obtained from a saddle-point evaluation of the functional integral [17] in Eq. (5) which becomes exact for the Gaussian VWF. ${ }^{3}$

Although the nonlinear sigma model (7) is easier to handle than the original Yang-Mills theory, its full nonperturbative treatment remains beyond analytical reach [25]. Nevertheless, the parametric enhancement of the action (7) by the factor $g_{b}^{-2}$ suggests that a useful approximation may be obtained from a saddle-point expansion of the functional integral over $U$. In order to render this

\footnotetext{
${ }^{2}$ Other variational and related Schwinger-Dyson equation studies on the basis of Gaussian trial states were carried out in gauge-fixed formulations [20,21].

${ }^{3}$ The gauge group of SU(N) Yang-Mills theory without matter is, strictly speaking, the coset $\mathrm{SU}(N) / Z_{N}$ since the center elements act trivially on the gauge fields. We refrain from making this restriction manifest, although it could be implemented in the action (7) and may become relevant, e.g., for the discussion of center vortices.
} 
approximation practical, however, one has to deal with the nonlocality of the bare action (7) which encumbers the identification and treatment of the saddle points. We will show in the following section that this can be efficiently accomplished by combining a renormalization group evolution of the bare action (which removes the explicit UV modes) with a subsequent derivative expansion. The result will be an approximately local soft-mode action for the IR fields.

\section{SOFT GRADIENT EXPANSION}

For the reasons outlined in the introduction, we are mainly interested in soft Yang-Mills amplitudes with external momenta $\left|\vec{p}_{i}\right|$ smaller than a typical hadronic scale $\mu$ (the lowest glueball mass, for example). This restricted focus permits us to recast the bare action (7) into a form which only retains soft field modes explicitly and which can be systematically approximated by a local Lagrangian. In the present section we describe the derivation and some useful features of this soft-mode action.

For proper choices of $G$ (see below) the dynamics (7) incorporates asymptotic freedom. Hence the bare coupling $g_{b}$ is small at the large cutoff scale $\Lambda_{\mathrm{UV}}$ where the theory is originally defined. The hard $U$ field modes with momenta $|\vec{k}|>\mu$ can therefore be integrated out of the functional integral (4) perturbatively, down to values of the infrared scale $\mu$ where the renormalized coupling $g(\mu)$ ceases to be much smaller than unity. In practice, this may for instance be done by Wilson's momentum-shell technique [26], after factorizing $U$ into contributions from high- and lowfrequency modes. To one-loop order, the resulting renormalization of the action just amounts to the replacement of the bare coupling $g_{b}$ by the running coupling $g(\mu)$. This was confirmed in Ref. [27] where the one-loop coupling was found to be

$$
g(\mu)=g_{b}+\frac{g_{b}^{3} N_{c}}{(2 \pi)^{2}} \ln \frac{\Lambda_{\mathrm{UV}}}{\mu}+O\left(g_{b}^{5}\right)
$$

(for $G(k)=k^{-1}$ at $k>\mu$ ). The scaling behavior of $g(\mu)$ makes asymptotic freedom explicit and reaffirms that the Gaussian VWF reproduces the qualitative UV behavior of Yang-Mills theory. In fact, Eq. (10) equals the one-loop Yang-Mills coupling up to a small correction factor 1/11 which could be avoided by allowing for an anisotropic component of $G^{-1}$ [17]. The one-loop integration over the high-momentum modes was found to be reliable down to $\mu \simeq 1.3 \mathrm{GeV}$ [27] which provides a useful benchmark for numerical estimates. In the proper $\mu$ range Eq. (7) turns into the renormalized soft-mode action

$$
\Gamma\left[U_{<}\right]=\frac{1}{4 g^{2}(\mu)} \int d^{3} x \int d^{3} y L_{<, i}^{a}(\vec{x}) G^{-1}(\vec{x}-\vec{y}) L_{<, i}^{b}(\vec{y}),
$$

where the subscript " $<$ " indicates that $U$ contains only $k<\mu$ modes.

The nonlocality of the action (11) is substantially weaker than that of the bare action (7) since the soft $U$ fields in the integrand vary too little to resolve details of $G^{-1}$ over distances smaller than $\mu^{-1}$. This observation can be turned into a controlled, local approximation scheme for the soft-mode action (11) by exploiting the fact that gradients of $U_{<}$are bounded by the IR gluon mass scale,

$$
\left\|\vec{\partial} U_{<}\right\|<\mu \text {. }
$$

Indeed, this bound suggests to expand the nonlocality of $G^{-1}$ into derivatives $\partial / \mu$ which will act upon $U_{<}$after partial integration. Using the isotropy of $G_{i j}^{-1}=G^{-1} \delta_{i j}$ (as mentioned above, an anisotropic component could be allowed in principle and would lead to somewhat more general expressions), one has

$$
\begin{aligned}
G^{-1}(\vec{x}-\vec{y})= & \mu\left[c_{0}+c_{1} \frac{\vec{\partial}_{x}^{2}}{\mu^{2}}+c_{2}\left(\frac{\vec{\partial}_{x}^{2}}{\mu^{2}}\right)^{2}\right. \\
& \left.+c_{3}\left(\frac{\vec{\partial}_{x}^{2}}{\mu^{2}}\right)^{3}+\ldots\right] \delta^{3}(\vec{x}-\vec{y}) .
\end{aligned}
$$

The dimensionless coefficients $c_{i}$ encode the lowmomentum behavior of $G^{-1}$ and may, e.g., be determined variationally. For our present purposes, however, it will be sufficient to adopt the standard expression $G^{-1}(\vec{k})=$ $\sqrt{\vec{k}^{2}+\mu^{2}}$ which approximates the solution of Schwinger-Dyson equations as well as variational estimates [20,21] and incorporates both asymptotic freedom and a dynamical mass gap. The corresponding $c_{i}$ can be read off directly from the Fourier transform

$$
\begin{aligned}
G^{-1}(\vec{x}-\vec{y}) & =\sqrt{-\vec{\partial}_{x}^{2}+\mu^{2}} \delta^{3}(\vec{x}-\vec{y}) \\
& =-\frac{1}{2 \pi^{2}} \frac{\mu^{2} K_{2}(\mu|x-y|)}{|x-y|^{2}}
\end{aligned}
$$

( $K_{2}$ is a McDonald function [28]), i.e. $c_{0}=1, c_{1}=-1 / 2$, $c_{2}=-1 / 8$, etc.

The combination of the above results leads to the intended reformulation of the nonlocal dynamics (7). As anticipated, the bilocal action density for the soft modes in Eq. (11) becomes a (quasi-) local Lagrangian and the action takes the familiar form

$$
\Gamma\left[U_{<}\right]=\int d^{3} x \mathcal{L}(\vec{x}) .
$$

Being a power series in the field gradients $\mu^{-1} \partial_{i} U_{<}$, the Lagrangian $\mathcal{L}(\vec{x})$ belongs to the class of generalized nonlinear sigma models. When expressed in terms of the Cartan-Maurer currents (8), it reads ${ }^{4}$

\footnotetext{
${ }^{4}$ The Lagrangian (16) could at once be generalized to arbitrary $G^{-1}$ by restoring the original $c_{i}$ dependence from Eq. (13).
} 


$$
\begin{aligned}
\mathcal{L}(\vec{x})= & -\frac{\mu}{2 g^{2}(\mu)} \operatorname{tr}\left\{L_{<, i}(\vec{x}) L_{<, i}(\vec{x})\right. \\
& +\frac{1}{2 \mu^{2}} \partial_{i} L_{<, j}(\vec{x}) \partial_{i} L_{<, j}(\vec{x}) \\
& \left.-\frac{1}{8 \mu^{4}} \partial^{2} L_{<, i}(\vec{x}) \partial^{2} L_{<, i}(\vec{x})+\ldots\right\} .
\end{aligned}
$$

In Eq. (16) we have omitted total derivatives $\Delta \mathcal{L}$ from the higher-order terms since they do not affect the field equations and generally have no influence on the saddle-point expansion. Nevertheless, they may generate nonvanishing surface terms due to infinite-action configurations. To lowest order,

$$
\Delta \mathcal{L}(\vec{x})=\frac{1}{8 g^{2}(\mu) \mu} \operatorname{tr}\left\{\partial^{2}\left[L_{<, i}(\vec{x}) L_{<, i}(\vec{x})\right]+\ldots\right\} .
$$

The gradient expansion in Eq. (16) is controlled by increasing powers of the parametrically small $\left(\partial U_{<} / \mu\right)^{2}$. For practical purposes it can therefore be reliably truncated, at an order which is determined by the desired accuracy of the approximation to the exact action. Below, we will be interested in specific field configurations (saddle points) which we are going to find explicitly. For those one may directly check a posteriori whether the full action is sufficiently well reproduced and, if not, include contributions from higher gradients. Based on such tests and a virial theorem (see Sec. VA), we found the truncation at $O\left((\partial / \mu)^{4}\right)$ to yield a generally sufficient approximation (at the few percent level) to the full action (11).

The first term in the Lagrangian (16) has the standard form of a 3-dimensional nonlinear $\sigma$-model (or principal chiral model). The second one, with four derivatives acting on $U_{<}$'s, is reminiscent of a similar term in the Skyrme model [29]. However, the identity

$$
\partial_{i} L_{j} \partial_{i} L_{j}=\frac{1}{2}\left[L_{i}, L_{j}\right]^{2}+\partial_{i} L_{j} \partial_{j} L_{i}
$$

shows that the four-derivative contribution to Eq. (16) contains, besides the commutator or Skyrme term, a part which qualitatively alters the character of the EulerLagrange equations. While the commutator would generate field equations of second order (see below), the piece without equivalent in the Skyrme model leads to additional fourth-order terms which allow for new types of solutions. (Several families of topological soliton solutions from the Lagrangian (16) will nevertheless resemble static Skyrmions ${ }^{5}$.)

We end this section by emphasizing that an analogous gradient expansion in terms of the original gauge fields $\vec{A}$

\footnotetext{
${ }^{5}$ It should be kept in mind, of course, that our $U$ field arises from Yang-Mills theory even without quarks, and that its physical interpretation (representing gauge fields of a fixed relative color orientation) differs from that in the Skyrme model where $U$ represents fluctuations around the chiral order parameter.
}

would require the (residual) gauge freedom to be completely fixed and thus give rise to all the associated conceptual and calculational complications. (Otherwise any "soft" gauge field could be turned into a "hard" one by a gauge transformation or, in other words, the "convergence" of the derivative expansion would be gauge dependent.) Hence the locality and structural simplicity of the Lagrangian (16) turns out to be a benefit of reformulating the dynamics in terms of gauge-invariant soft-mode fields.

\section{SADDLE-POINT EXPANSION}

Our next task will be the development of a saddle-point (or, more specifically, steepest descent) expansion for the functional integral over the soft modes,

$$
Z=\int D U_{<} \exp \left(-\Gamma\left[U_{<}\right]\right)
$$

where the vacuum overlap $Z$ serves as a prototype for similar integrals in other amplitudes. The building blocks of this expansion are the IR saddle-point fields $\bar{U}_{i}(\vec{x})$, i.e. the local minima of the soft-mode action (15). Their identification is simplified by the fact that all finite-action $U$ fields fall into disjoint topological classes (cf. Sec. V B and below). Indeed, fields of different topological charge-for now summarily denoted by $Q$ - cannot be continuously deformed into each other and the local variation of the action may therefore be performed in each topological sector separately. This amounts to solving the saddle-point equations

$$
\left.\frac{\delta \Gamma\left[U_{<}\right]}{\delta U_{<}(\vec{x})}\right|_{U_{<}=\bar{U}_{i}^{(Q)}}=0
$$

at fixed $Q$.

The saddle-point expansion for the vacuum overlap $Z$ can then be assembled by adding the contributions from all relevant solutions $\bar{U}_{i}^{(Q)}$ of Eq. (20). To leading order,

$$
Z \simeq \sum_{Q \in Z, i} F_{i}\left[\bar{U}_{i}^{(Q)}\right] \exp \left(-\Gamma\left[\bar{U}_{i}^{(Q)}\right]\right)
$$

where the preexponential factors $F_{i}$ are generated by zeromode contributions which typically arise if continuous symmetries of the action are broken by the $\bar{U}$. The sum over the saddle points, labeled by $i$, symbolically includes integrals with the appropriate measure whenever the saddle points come in continuous families. Functional integrals of more complex amplitudes, for example gluonic Green functions, are obtained from the same saddle points by differentiating $Z$ with respect to suitably implemented sources. Higher-order corrections to Eq. (21) can be sys- 
tematically calculated from (nondegenerate) fluctuations around the solutions $\bar{U}_{i}^{(Q)}$. The reliability of the leading order increases with the action values at the saddle points because $\Gamma[\bar{U}] \gg 1$ prevents their contributions from being rendered insignificant by the fluctuations of $O(1)$ around them. In our case, compliance with this criterion is parametrically enhanced ${ }^{6}$ by the overall factor $g^{-2}(\mu) \gg 1$ (for $\mu \gtrsim 1.3-1.5 \mathrm{GeV}$ ) in the Lagrangian (16).

For the explicit solution of the saddle-point Eq. (20), as well as for part of our general analysis below, it is practically indispensable to parametrize the $\mathrm{SU}(N)$ elements in terms of their $N^{2}-1$ independent degrees of freedom. We will use the exponential representation

$$
U_{<}(\vec{x})=\exp \left[\phi(\vec{x}) \hat{n}^{a}(\vec{x}) \frac{\tau^{a}}{2 i}\right]
$$

for this purpose. In Eq. (22) the coefficient vector of the Lie-algebra generators is decomposed into its length $\phi$ and its direction $\hat{n}^{a}$ with $\hat{n}^{a} \hat{n}^{a}=1$ which parametrizes the coset $\mathrm{SU}(N) / H$ with respect to the Cartan subgroup $H$.
For simplicity, we will also specialize the following discussion to $N=2$. Our soft-mode Lagrangian (16) can then be rewritten in terms of the unit-vector field $\hat{n}^{a}$ and the spin-0 field $\phi$ as

$$
\mathcal{L}(U)=\mathcal{L}_{2 d}(\phi, \hat{n})+\mathcal{L}_{4 d}(\phi, \hat{n})
$$

where, for the reasons discussed in the paragraph below Eq. (17), all terms of the gradient expansion with up to four derivatives on the $U$ fields are retained. The two-derivative part $\mathcal{L}_{2 d}$, i.e. the standard nonlinear $\sigma$-model, becomes

$$
\mathcal{L}_{2 d}=\frac{\mu}{2^{2} g^{2}(\mu)}\left[\left(\partial_{i} \phi\right)^{2}+2(1-\cos \phi)\left(\partial_{i} \hat{n}^{a}\right)^{2}\right]
$$

while the four-derivative contributions turn into

$$
\begin{aligned}
\mathcal{L}_{4 d}= & \frac{1}{2^{3} g^{2}(\mu) \mu}\left[\left(\partial^{2} \phi-\sin \phi\left(\partial_{i} \hat{n}^{a}\right)^{2}\right)^{2}+2(1+\cos \phi)\left(\partial_{i} \phi \partial_{i} \hat{n}^{a}\right)^{2}+2(1-\cos \phi)\left(\varepsilon_{i j k} \partial_{j} \phi \partial_{k} \hat{n}^{a}\right)^{2}\right. \\
& \left.+4 \sin \phi \partial_{j} \phi \partial_{j} \hat{n}^{a} \partial^{2} \hat{n}^{a}+2(1-\cos \phi)\left(\varepsilon^{a b c} \hat{n}^{b} \partial^{2} \hat{n}^{c}\right)^{2}+2(1-\cos \phi)^{2}\left(\varepsilon^{a b c} \partial_{i} \hat{n}^{b} \partial_{j} \hat{n}^{c}\right)^{2}\right] .
\end{aligned}
$$

The above expressions show that $\mathcal{L} \geq 0$, as required for the existence of the functional integral (19). The same remains true if higher orders of the derivative expansion (16) are included (as long as $\partial U_{<} / \mu<1$, of course). For our subsequent analysis of generic saddle-point solution properties, including their scaling behavior (cf. Sec. V), it will prove useful that both $\mathcal{L}_{2 d}$ and $\mathcal{L}_{4 d}$ are also individually non-negative.

By varying the action (15) of the Lagrangian (23) with respect to $\phi$ and $\hat{n}^{a}$, the general saddle-point Eq. (20) turns into a nonlinear system of four coupled partial differential equations. For $\phi$ one directly obtains

$$
\begin{aligned}
\left(4 g^{2} \mu\right) \frac{\delta \Gamma[\phi, \hat{n}]}{\delta \phi(\vec{x})}= & \partial^{4} \phi-2 \partial^{2} \phi\left(\partial_{i} \hat{n}^{a}\right)^{2}-4 \partial_{i} \phi \partial_{i} \partial_{k} \hat{n}^{a} \partial_{k} \hat{n}^{a}-4 \cos \phi\left(\partial_{i} \partial_{j} \phi \partial_{i} \hat{n}^{a}+\partial_{j} \phi \partial^{2} \hat{n}^{a}+\partial_{i} \phi \partial_{i} \partial_{j} \hat{n}^{a}\right) \partial_{j} \hat{n}^{a} \\
& +2 \sin \phi\left[\left(\partial_{i} \phi \partial_{i} \hat{n}^{a}\right)^{2}-(1-\cos \phi)\left(\partial_{i} \hat{n}^{a} \partial_{j} \hat{n}^{a}\right)^{2}-2 \partial_{i} \hat{n}^{a} \partial_{i} \partial^{2} \hat{n}^{a}-\left(\partial_{i} \partial_{j} \hat{n}^{a}\right)^{2}+\left(\partial_{i} \hat{n}^{a}\right)^{2}\left(\partial_{j} \hat{n}^{b}\right)^{2}\right] \\
& -\sin \phi \cos \phi\left(\partial_{i} \hat{n}^{a}\right)^{2}\left(\partial_{j} \hat{n}^{b}\right)^{2}-\sin \phi\left(\delta^{a b}+\hat{n}^{a} \hat{n}^{b}\right) \partial^{2} \hat{n}^{a} \partial^{2} \hat{n}^{b}-2 \mu^{2}\left[\partial^{2} \phi-\sin \phi\left(\partial_{i} \hat{n}^{a}\right)^{2}\right]=0 .
\end{aligned}
$$

The three equations for the components of the vector field, on the other hand, have to be derived by a constrained variation whose task it is to preserve the unit length of $\hat{n}$. As a consequence, they can be cast into the succinct form

\footnotetext{
${ }^{6}$ This enhancement is somewhat weaker than in the bare action (7) since $g(\mu) \geq g_{b}$.
}

$$
\left(\delta^{a b}-\hat{n}^{a} \hat{n}^{b}\right) \frac{\delta \Gamma[\phi, \hat{n}]}{\delta \hat{n}^{a}(\vec{x})}=0
$$

where the projection operator ensures that the action is varied only by those $\delta \hat{n}^{a}$ which remain orthogonal to $\hat{n}^{a}$. The evaluation of the functional derivative yields 


$$
\begin{aligned}
\left(2 g^{2} \mu\right) \frac{\delta \Gamma[\phi, \hat{n}]}{\delta \hat{n}^{a}(\vec{x})}= & -(1-\cos \phi)\left(\partial^{2} \hat{n}^{a} \hat{n}^{c} \partial^{2} \hat{n}^{c}\right)+\partial_{i}\left\{\sin \phi \partial^{2} \phi \partial_{i} \hat{n}^{a}-\sin \phi \partial_{i} \phi \partial^{2} \hat{n}^{a}-2 \cos \phi \partial_{i} \phi \partial_{j} \phi \partial_{j} \hat{n}^{a}\right. \\
& +\cos \phi \partial_{i} \phi \partial_{k} \phi \partial_{k} \hat{n}^{a}+\sin \phi \partial_{i} \partial_{k} \phi \partial_{k} \hat{n}^{a}+\sin \phi \partial_{k} \phi \partial_{i} \partial_{k} \hat{n}^{a}+\sin \phi \partial_{i} \phi\left(\partial^{2} \hat{n}^{a}-\hat{n}^{a} \hat{n}^{c} \partial^{2} \hat{n}^{c}\right) \\
& +(1-\cos \phi)\left[\partial_{i} \partial^{2} \hat{n}^{a}-\hat{n}^{c} \partial_{i} \hat{n}^{a} \partial^{2} \hat{n}^{c}-\hat{n}^{a} \partial_{i} \hat{n}^{c} \partial^{2} \hat{n}^{c}-\hat{n}^{a} \hat{n}^{c} \partial_{i} \partial^{2} \hat{n}^{c}-\left(\partial_{j} \phi\right)^{2} \partial_{i} \hat{n}^{a}-2 \mu^{2} \partial_{i} \hat{n}^{a}\right] \\
& \left.-\sin ^{2} \phi \partial_{i} \hat{n}^{a}\left(\partial_{i} \hat{n}^{c}\right)^{2}-2(1-\cos \phi)^{2}\left[\partial_{i} \hat{n}^{a}\left(\partial_{j} \hat{n}^{c}\right)^{2}-\partial_{i} \hat{n}^{c} \partial_{j} \hat{n}^{c} \partial_{j} \hat{n}^{a}\right]\right\} .
\end{aligned}
$$

The saddle-point Eqs. (26) and (27) are independent of the coupling $g$ which enters the action only through an overall factor $g^{-2}$. In general, the saddle-point solutions have to be found numerically. However, we will demonstrate below that several nontrivial analytical solutions exist and that further important solution classes with a rather high degree of symmetry can be obtained by solving much simpler field equations.

Moreover, essential qualitative solution properties can be derived without solving the saddle-point equations at all (cf. Sec. V) and will provide guidance for applications of the expansion (21). Each topological charge sector contains at least one action minimum, for example, i.e. one solution of Eqs. (26) and (27). The formidable task of finding and including all of them would render the saddle-point expansion practically useless. Fortunately, however, this turns out to be unnecessary. Below we will establish lower bounds on the action of the saddle points which are monotonically increasing functions of the absolute value of their topological charges. Hence contributions from saddle points in high charge sectors can generally be neglected.

\section{GENERAL PROPERTIES OF THE SADDLE-POINT SOLUTIONS}

Before actually solving the saddle-point Eqs. (26) and (27) explicitly in Secs. VI and VII, we will now interject the derivation of a few useful generic results which clarify the topological structure and stability properties of the solutions.

\section{A. Virial theorem}

In order to analyze the scaling behavior of the extended saddle-point solutions and to establish the underlying virial theorem, we define the scaled fields

$$
\phi_{\lambda}:=\phi(\lambda \vec{x}), \quad \hat{n}_{\lambda}^{a}:=\hat{n}^{a}(\lambda \vec{x})
$$

for real $\lambda \neq 0$ and note that $\mu$ is the only mass scale in the field equations (26) and (27). The latter implies that solutions of the equations with scaled parameter $\mu \rightarrow \lambda^{-1} \mu$ can be obtained by rescaling the original solutions $(\bar{\phi}, \hat{n})$ to $\left(\bar{\phi}_{\lambda}, \hat{n}_{\lambda}\right)$.

In the following, however, we keep $\mu$ fixed. Localized, scale-transformed solutions then cease to solve the field equations, and this fact together with two basic properties of the Lagrangian (23) can be turned into a virial theorem.
Its derivation rests on the relation between the actions of scaled and unscaled fields (as long as they stay finite), which can be read off from the 2- and 4-derivative parts of the Lagrangian (23) as

$$
\begin{aligned}
\Gamma(\lambda) & :=\Gamma\left[\phi_{\lambda}, \hat{n}_{\lambda}\right]=\Gamma_{2 d}(\lambda)+\Gamma_{4 d}(\lambda) \\
& =\frac{1}{\lambda} \Gamma_{2 d}(1)+\lambda \Gamma_{4 d}(1) .
\end{aligned}
$$

A second relevant property of the action is its strict positivity for extended, i.e. not translationally invariant field configurations (cf. Sec. IV),

$$
\Gamma_{2 d}(1), \Gamma_{4 d}(1)>0 .
$$

The remaining step is to specialize the fields under consideration to the saddle-point solutions. Since those extremize the action under arbitrary small variations - which of course include infinitesimal scale transformations - one immediately deduces $d \Gamma(\lambda) /\left.d \lambda\right|_{\lambda=1}=0$ and consequently the virial theorem

$$
\Gamma_{2 d}(1)=\Gamma_{4 d}(1) .
$$

Equation (32) exhibits the pivotal role of the fourderivative terms $\Gamma_{4 d}$ in guaranteeing the stability of the saddle-point solutions: for $\Gamma_{4 d}=0$ the remaining nonlinear $\sigma$-model action would be minimized by sending $\lambda \rightarrow$ $\infty$ [cf. Equation (30)], i.e. by the scale collapse of the solutions which is a consequence of Derrick's theorem [30]. Our truncation of the gradient expansion (16) at $O\left(\left(\partial U_{<} / \mu\right)^{2}\right)$ therefore turns out to be the minimal approximation which supports localized, stable soliton solutions ${ }^{7}$. Furthermore,

$$
\left.\frac{d^{2} \Gamma(\lambda)}{d \lambda^{2}}\right|_{\lambda=1}=2 \Gamma_{2 d}(1) \geq 0
$$

\footnotetext{
${ }^{7}$ Since the signs of the higher-order terms in the derivative expansion (16) alternate, one might suspect that their contributions could destabilize extended solutions. This possibility would arise only after an inconsistent truncation of the gradient expansion, however: if a localized solution is small enough to be significantly affected by higher-derivative terms, the latter would have to be added to the field equations in the first place. (A somewhat analogous situation is encountered in ColemanWeinberg potentials at one-loop order: their physically relevant local minima are stable only as long as fluctuations remain small, i.e. as long as the underlying truncation of the loop expansion is justified [31].)
} 
implies that the scaling extrema are indeed action minima and that solutions with $\Gamma_{2 d}(1)=0$ are points of inflection.

It is worth noting that the coexistence of terms with different numbers of derivatives in the Lagrangian (16), and thus ultimately the virial theorem (32) and the emergence of stable solutions, have their origin in the presence of the mass scale $\mu$ which reflects the short-wavelength quantum fluctuations integrated out in Sec. III. This situation is reminiscent of the classical Yang-Mills instanton solutions whose characteristic size scale is likewise generated by quantum fluctuations.

To summarize, the virial theorem primarily ensures that the extended solutions of Eqs. (26) and (27) are stable against scale transformations. In our context this is indispensable since unstable solutions would prohibit a consistent saddle-point expansion. As a useful side benefit, the virial theorem provides stringent constraints on the numerical solutions of the saddle-point equations.

\section{B. Topology}

As a three-dimensional principal chiral model with stabilizing higher-derivative terms, the soft-mode Lagrangian (23) is expected to sustain topological soliton solutions. In the present section we discuss three topological charges (i.e. invariants) which such solutions and more general continuous $U$ fields may carry [32].

The most fundamental homotopy classification arises from the fact that all $U$ fields with a finite action $\Gamma$ [based on any truncation of the Lagrangian (16)] have to approach the same constant as $|\vec{x}| \rightarrow \infty$. In analogy with the stereographic projection, this boundary condition compactifies the domain of the fields to a three-sphere $S_{s p}^{3}$, i.e. all $U$ 's with finite $\Gamma$ describe continuous maps from $S_{s p}^{3}$ into the "topologically active" part of the group manifold. For $\mathrm{SU}(N)$ the latter is the trivially embedded subgroup $\mathrm{SU}(2) \sim S_{G}^{3}$. The resulting maps $S_{s p}^{3} \rightarrow S_{G}^{3}$ fall into disjoint homotopy classes which form the elements of the homotopy group $\pi_{3}\left(S^{3}\right)=\mathbb{Z}$ and are characterized by a topological degree or charge

$$
Q[U]=\frac{1}{24 \pi^{2}} \int d^{3} x \varepsilon_{i j k} \operatorname{tr}\left\{U^{\dagger} \partial_{i} U U^{\dagger} \partial_{j} U U^{\dagger} \partial_{k} U\right\} .
$$

(The integrand can be shown to be a total derivative, as expected from a topological charge density.) For finiteaction fields $Q \in \mathbb{Z}$. In terms of the $(\phi, \hat{n})$ parametrization (22) for $U \in \mathrm{SU}(2)$, Eq. (34) reduces to

$Q[\phi, \hat{n}]=\frac{1}{2^{4} \pi^{2}} \int d^{3} x(\cos \phi-1) \varepsilon_{i j k} \varepsilon^{a b c} \partial_{i} \phi \hat{n}^{a} \partial_{j} \hat{n}^{b} \partial_{k} \hat{n}^{c}$.

Two additional topological quantum numbers are carried solely by the $\hat{n}$-field component of $U$. The first one is the homotopy degree of the map from the space boundary $S_{\infty}^{2}($ at $|\vec{x}| \rightarrow \infty)$ into the unit sphere $S_{\hat{n}}^{2}$ on which $\hat{n}^{a}$ takes values. Continuous maps of this sort are classified by the elements of the homotopy group $\pi_{2}\left(S^{2}\right)=\mathbb{Z}$. (The same topology generates the magnetic charge of the Wu-Yang monopole [33].) An explicit integral representation of the corresponding degree is

$$
q_{m}[\hat{n}]=\frac{1}{8 \pi} \int_{\partial R^{3}} d \sigma_{i} \varepsilon_{i j k} \varepsilon^{a b c} \hat{n}^{a} \partial_{j} \hat{n}^{b} \partial_{k} \hat{n}^{c},
$$

where the integral extends over the closed surface $\partial R^{3}=$ $S_{\infty}^{2}$. As expected, $q_{m}$ is manifestly $\phi$-independent.

The third topological invariant owes its existence to the fact that all $\hat{n}$ fields of finite action are required to approach a constant unit vector at spacial infinity. As above, this requirement compactifies $R^{3}$ into $S_{s p}^{3}$ and thereby turns $\hat{n}(\vec{x})$ into a continuous map $S_{s p}^{3} \rightarrow S_{\hat{n}}^{2}$. Such maps carry a Hopf charge $q_{H}$ which labels the elements of the homotopy group $\pi_{3}\left(S^{2}\right)=\mathbb{Z}$, i.e. the Hopf bundle [34]. A useful integral representation for $q_{H}$ can be constructed by exploiting the local isomorphism between nonlinear $\mathrm{O}(3)$ and $C P(1)$ fields which maps $\hat{n}^{a}$ into a complex 2-component field $\chi=\left(\chi_{1}, \chi_{2}\right)$ with unit modulus $\chi^{\dagger} \chi=\chi_{1}^{*} \chi_{1}+$ $\chi_{2}^{*} \chi_{2}=1$ by indentifying $\hat{n}^{a}=\chi^{\dagger} \tau^{a} \chi$. In terms of $\chi$, the Hopf charge of $\hat{n}$ takes the explicit form

$$
q_{H}[\hat{n}(\chi)]=\frac{1}{4 \pi^{2}} \int d^{3} x \varepsilon_{i j k}\left(\chi^{\dagger} \partial_{i} \chi\right)\left(\partial_{j} \chi^{\dagger} \partial_{k} \chi\right) .
$$

(A local integral representation for $q_{H}$ directly in terms of the $\hat{n}$-field does not exist.) In contrast to the topological charges $Q$ and $q_{m}$ which are of winding-number type, the Hopf invariant $q_{H}$ is a linking number. The underlying topological structure allows for and classifies the link and knot solutions to be encountered in Sec. VIC.

Finally, we recall that the $U$ field topology - as summarized in the conserved quantum numbers $Q, q_{m}$, and $q_{H}-$ classifies not only the saddle-point solutions but the much larger space of continuous field configurations with finite and in some cases even infinite (see below) action.

\section{Bogomol'nyi bound}

The distribution of the solutions to Eqs. (26) and (27) over a (denumerably) infinite set of topological charge sectors allows for the existence of more saddle points than could be handled in practical applications of the expansion (21). Hence additional criteria are required to select the most relevant saddle points in a controlled fashion. Such criteria can indeed be established, in the form of lower bounds on the action which are monotonically increasing functions of the absolute topological charge values. In the present section we obtain the action bound for fields which carry finite $Q$ values. A similar bound for fields with nonvanishing Hopf charge will be given in Sec. VIC. The Boltzmann weight factor $\exp (-\Gamma)$ then ensures that contributions from saddle points with higher topological quantum numbers are increasingly suppressed in functional integrals and can be systematically neglected. 
The lower bound on the action of the Lagrangian (23) for any field $U$ with a well-defined degree (34) can be derived from the Lie-algebra valued expression

$$
M_{i j}=a \partial_{i} L_{j}+b \varepsilon_{i j k} L_{k}
$$

where $a, b \in \mathbb{R}$ and $L_{i}$ are the components of the CartanMaurer one-form (8). With the help of the Maurer-Cartan identity

$$
\partial_{i} L_{j}-\partial_{j} L_{i}=\left[L_{j}, L_{i}\right]
$$

one then obtains for its square

$$
M_{i j} M_{i j}=a^{2} \partial_{i} L_{j} \partial_{i} L_{j}-2 a b \varepsilon_{i j k} L_{i} L_{j} L_{k}+2 b^{2} L_{i} L_{i}
$$

which obeys the basic inequality

$$
\operatorname{tr}\left\{M_{i j} M_{i j}\right\} \leq 0 .
$$

(Recall from Eq. (8) that the $L_{i}$ are expanded into antiHermitian generators.)

After specializing the bound (41) to $b=1 / \sqrt{2}$ and $a= \pm 1 /(\sqrt{2} \mu)^{8}$, multiplying by $-\mu /\left(2 g^{2}\right)$, integrating over $\vec{x}$ and using the integral representation (34) for $Q$, one arrives at

$$
\begin{aligned}
& -\frac{\mu}{2 g^{2}(\mu)} \int d^{3} x \operatorname{tr}\left\{L_{i} L_{i}+\frac{1}{2 \mu^{2}} \partial_{i} L_{j} \partial_{i} L_{j}\right\} \\
& \geq \mp \frac{12 \pi^{2}}{g^{2}(\mu)} Q[U] .
\end{aligned}
$$

The more stringent of these inequalities results from the lower (upper) sign on the right-hand side if $Q>0(Q<0)$. Their left-hand side is equal to the action which the first two terms ${ }^{9}$ of the Lagrangian (16) generate. Hence the expressions (42) for both signs combine into an inequality of Bogomol'nyi type,

$$
\Gamma[U] \geq \frac{12 \pi^{2}}{g^{2}(\mu)}|Q[U]| .
$$

This is the desired lower bound on the action of any $U$ field with well-defined homotopy degree $Q$. Its saturation requires the associated minimal-action fields to solve the Bogomol'nyi-type equation

$$
\partial_{i} L_{j}=\mp \mu \varepsilon_{i j k} L_{k}
$$

where the lower (upper) sign again refers to $Q>0(Q<$ 0 ). Equation (44) can be considered as an analog of the

\footnotetext{
${ }^{8}$ Different choices for the coefficients $c_{0,1}$ in Eq. (13) could be accomodated by modifying the values of $a$ and $b$ and would result in a different factor in front of $|Q|$ in the Bogomol'nyi bound.

${ }^{9}$ For special purposes, e.g. for the discussion of infinite-action solutions, it may be useful to maintain the surface term (17) in $\Gamma$. After specializing to $b=1 / \sqrt{2}$ and $a= \pm 1 /(\sqrt{2} \mu)$, the obvious identity $\operatorname{tr}\left\{\partial_{i} L_{j} \partial_{i} L_{j}\right\}=\operatorname{tr}\left\{\frac{1}{2} \partial^{2}\left(L_{i} L_{i}\right)-L_{i} \partial^{2} L_{i}\right\}$ then results in $\Gamma[U]=-\frac{\mu}{2 g^{2}(\mu)} \int d^{3} x \operatorname{tr}\left\{L_{i}^{2} L_{i}-\frac{1}{2 \mu^{2}} L_{i} \partial^{2} L_{i}\right\} \geq \mp \frac{12 \pi^{2}}{g^{2}(\mu)} Q[U]+$ $\int d^{3} x \Delta \mathcal{L}(\vec{x})$.
}

self-(anti)-duality equation in Yang-Mills theory. Its solutions form a subset of those of the more general field equations (26) and (27) and have the characteristic property that their rescaled Cartan-Maurer currents $\tilde{L}_{i}:=$ $\mp L_{i} /(2 i \mu)$ in any $Q$-sector generate the SU(2) Lie algebra, i.e.

$$
\left[\tilde{L}_{i}, \tilde{L}_{j}\right]=i \varepsilon_{i j k} \tilde{L}_{k} .
$$

Translationally invariant solutions (cf. Sec. VI A), in particular, have $\tilde{L}_{i}=0$ and therefore trivially saturate the bound in the $Q=0$ sector. It remains to be seen whether nontrivial solutions in sectors with larger $|Q|$ exist as well ${ }^{10}$.

The large factor multiplying $|Q|$ in the bound (43) indicates that contributions from saddle points with higher $|Q|$ are strongly suppressed and seem safely negligible in amplitudes which receive nonvanishing contributions from the $Q=0$ sector. However, one should keep in mind that even saddle points with extremely small "fugacities" can sometimes have a decisive physical impact. A case in point are the crucial monopole contributions to the partition function of the $2+1$ dimensional Yang-Mills-Higgs model [36]. A detailed discussion of the $|Q| \neq 0$ solutions and their physical interpretation will be the subject of Secs. VID, VIE, and VII.

\section{IMPORTANT SADDLE-POINT SOLUTION CLASSES}

In the following sections we are going to solve the four saddle-point Eqs. (26) and (27) explicitly. As already mentioned, our main focus will be on solutions with a relatively high amount of symmetry since their generally smaller action values imply a stronger impact on most amplitudes. As a side benefit, these solutions can often be obtained either analytically or with moderate numerical effort.

\section{A. Translationally invariant solutions}

The simplest solutions of the saddle-point Eqs. (26) and (27) are the $\vec{x}$-independent matrices

$$
U_{c}=\exp \left[\phi_{c} \hat{n}_{c}^{a} \frac{\tau^{a}}{2 i}\right]=\text { const, }
$$

where $\phi_{c}$ and $\hat{n}_{c}^{a}$ are both constant. These solutions form the complete vacuum manifold of the dynamics (23), i.e. the set of all fields which attain the absolute action minimum

$$
\Gamma\left[U_{c}\right]=0 .
$$

Because of a redundancy in the parametrization (22), the

\footnotetext{
${ }^{10}$ The analogous bound in the Skyrme model for $Q=1$ can only be saturated on a hyperspherical domain $S^{3}$, for example [35].
} 
subset of vacua in the center of the gauge group is completely $\hat{n}^{a}$-independent:

$$
\phi_{c, k}=2 k \pi, \quad U_{c, k}=(-1)^{k} .
$$

In addition, those are the only vacua which do not break the global U(2) symmetry of the Lagrangian (23) spontaneously. A glance at the integral representation (35) shows that none of the $U_{c}$ carries topological charges $Q \neq 0$.

\section{B. Constant- $\hat{\boldsymbol{n}}$ solutions}

Any constant vector $\hat{n}^{a}$ solves the saddle-point Eqs. (27) identically and reduces the remaining one, Eq. (26) for $\phi$, to the linear field equation

$$
\partial^{2}\left(\partial^{2} \phi-2 \mu^{2} \phi\right)=0 .
$$

Obviously, the solutions of this equation come in families which differ by an additive constant and have degenerate action values. (This trivially ensures periodicity in $\phi$.) Alternatively, Eq. (49) and the action of its solutions can be derived from the reduced Lagrangian ${ }^{11}$

$$
\mathcal{L}^{(\hat{n}=c)}=\frac{1}{2^{3} g^{2}(\mu) \mu}\left[\left(\partial^{2} \phi\right)^{2}+2 \mu^{2}\left(\partial_{i} \phi\right)^{2}\right] .
$$

The presence of the 4th-order term in Eq. (49) allows for solution types without equivalent in Skyrme models. (This is because the commutator or Skyrme term in Eq. (18) produces a scale-free Laplace equation for $\phi$ whose regular finite-action solutions on $\mathbb{R}^{3}$ are constants.) A glance at the integrals (35)-(37) shows that solutions with constant $\hat{n}$ do not carry any topological quantum numbers, i.e.

$$
Q^{(\hat{n}=c)}=q_{m}^{(\hat{n}=c)}=q_{H}^{(\hat{n}=c)}=0 .
$$

All solutions of the linear field equation (49) can be constructed by standard Green function techniques. The perhaps most straightforward method involves folding the static Klein-Gordon propagator

$$
\Delta(\vec{x}-\vec{y} ; m)=-\int \frac{d^{3} k}{(2 \pi)^{3}} \frac{e^{i \vec{k}(\vec{x}-\vec{y})}}{\vec{k}^{2}+m^{2}}=-\frac{1}{4 \pi} \frac{e^{-m|\vec{x}-\vec{y}|}}{|\vec{x}-\vec{y}|}
$$

(for $\vec{x} \neq \vec{y}$ ) with a "scalar potential" $\Phi$ which is defined to be a solution of the Laplace equation, $\partial^{2} \Phi=0$, and acts as the inhomogeneity of the static Klein-Gordon equation

$$
\partial^{2} \phi-2 \mu^{2} \phi=\Phi .
$$

(Obviously, the field equation (49) is recovered from Eq. (53) by applying the Laplacian on both sides.) Since the potential $\Phi$ plays the role of a source for the $\phi$ field, inversion of the Klein-Gordon operator yields the general solution

\footnotetext{
${ }^{11}$ Although this Lagrangian is bilinear in $\phi$, its contributions to $Z$ remain nontrivial since the nonlinear Haar measure generates interactions among the $\phi$ modes.
}

$$
\phi(\vec{x})=\int d^{3} y \Delta(\vec{x}-\vec{y} ; \sqrt{2} \mu) \Phi(\vec{y}) .
$$

Of course, the regular finite-action solutions form but a small subset of those contained in Eq. (54).

Spherically symmetric solutions can be derived more directly by discarding the angular dependence of $\phi$, i.e. by substituting the ansatz $\phi(r)$ with $r \equiv|\vec{x}|$ into Eq. (49) and ignoring for the moment potential singularities at the origin. This yields the radial equation

$$
r \phi^{\prime \prime \prime \prime}+4 \phi^{\prime \prime \prime}-2 \mu^{2}\left(r \phi^{\prime \prime}+2 \phi^{\prime}\right)=0
$$

(derivatives with respect to $r$ are denoted by a prime) whose four linearly independent solutions can be found analytically:

$$
\begin{gathered}
\phi_{1}=c, \quad \phi_{2}=\frac{1}{\sqrt{2} \mu r}, \\
\phi_{3}=\frac{e^{+\sqrt{2} \mu r}}{\sqrt{2} \mu r}, \quad \phi_{4}=\frac{e^{-\sqrt{2} \mu r}}{\sqrt{2} \mu r} .
\end{gathered}
$$

Hence the subset of regular finite-action solutions has the form

$$
\bar{\phi}^{(\hat{n}=c)}(r)=c_{1}+\frac{c_{2}}{\sqrt{2} \mu r}\left(1-e^{-\sqrt{2} \mu r}\right) .
$$

The associated potential

$$
\bar{\Phi}^{(\hat{n}=c)}(r)=\left(\partial^{2}-2 \mu^{2}\right) \bar{\phi}^{(\hat{n}=c)}(r)=-2 \mu^{2}\left(c_{1}+\frac{c_{2}}{\sqrt{2} \mu r}\right)
$$

shows that the expression (57) in fact solves a generalization of the homogeneous field equation (49), with an additional delta-function singularity at the origin. Equation (57) is therefore a solution everywhere except at $\vec{x}=0$ and, strictly speaking, one of its Green functions. A representative of this solution class is drawn in Fig. 1.

After insertion into the action (15), based on the Lagrangian (23), and use of the virial theorem (32) one finds for the solutions (57)

$$
\Gamma\left[\phi^{(\hat{n}=c)}, \hat{n}_{c}\right]=\frac{2 \pi \mu}{g^{2}(\mu)} \int_{0}^{\infty} d r\left(r \phi^{(\hat{n}=c) \prime}\right)^{2}=\frac{\pi}{\sqrt{2}} \frac{c_{2}^{2}}{g^{2}(\mu)} .
$$

This action is not subject to topological bounds and reaches the absolute minimum $\Gamma=0$ at $c_{2}=0$ where the constant $-\hat{n}$ solutions turn into the translationally invariant vacua of Sec. VI A. [In contrast to the center elements (48), however, the value of $\phi$ remains unrestricted here.] Hence constant- $\hat{n}$ solutions with small action values can have a strong impact on low-energy amplitudes [15].

\section{Faddeev-Niemi type knot solutions}

In addition to the translationally invariant saddle points there exist less trivial solutions of the field equations (26) 


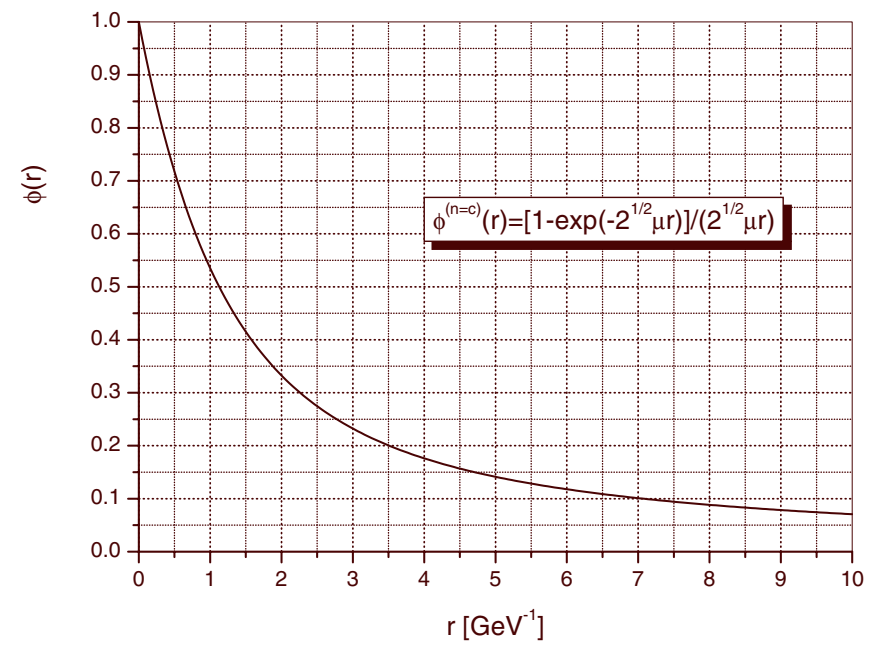

FIG. 1 (color online). An example of the $\hat{n}=$ const solution class. As in all following figures, we display the solution at $\mu=$ $1 \mathrm{GeV}$. Other values of $\mu$ can be reached by scaling the $r$ axis. Our conventions for the periodicity interval of $\phi$ restrict the initial value to $\phi(0) \in] 0,2 \pi]$.

and (27) with constant $\phi$ fields. Among them, the most intriguing class has the general form

$$
\phi_{c 2, k}=(2 k+1) \pi, \quad U_{c 2, k}(\vec{x})=(-1)^{k} i \tau^{a} \hat{n}^{a}(\vec{x}),
$$

which satisfies Eq. (26) identically and carries no topological charge $Q$. In fact, the nontrivial topology of $U_{c 2}$ (as that of any other constant- $\phi$ field configuration) has to reside exclusively in the $\vec{x}$-dependence of its $\hat{n}$ field, governed by the ( $k$-independent) equation

$$
\begin{array}{r}
\varepsilon^{a b c} \partial_{i}\left[\hat { n } ^ { b } \left(2 \mu^{2} \partial_{i} \hat{n}^{c}+2\left(\partial_{j} \hat{n}^{d}\right)^{2} \partial_{i} \hat{n}^{c}-4 \partial_{j} \hat{n}^{c} \partial_{i} \hat{n}^{d} \partial_{j} \hat{n}^{d}\right.\right. \\
\left.\left.-\partial^{2} \partial_{i} \hat{n}^{c}\right)\right]=0 .
\end{array}
$$

This field equation follows from the general saddle-point equation (27) by substituting $\phi_{c 2 . k}$ and plays the role of a (static) continuity equation for the conserved $\mathrm{O}(3)$ current. Alternatively, it can be obtained by directly varying the reduced Lagrangian

$$
\begin{aligned}
\mathcal{L}^{\left(\phi_{c 2}\right)}(\vec{x})= & \frac{\mu}{g^{2}(\mu)}\left[\left(\partial_{i} \hat{n}^{a}\right)^{2}+\frac{1}{\mu^{2}}\left(\varepsilon^{a b c} \partial_{i} \hat{n}^{b} \partial_{j} \hat{n}^{c}\right)^{2}\right. \\
& \left.+\frac{1}{2 \mu^{2}}\left(\varepsilon^{a b c} \hat{n}^{b} \partial^{2} \hat{n}^{c}\right)^{2}\right],
\end{aligned}
$$

which is the specialization of Eq. (23) to $\phi_{c 2 . k}$ and reproduces the action (15) for the $U_{c 2, k}$.

Remarkably, the Lagrangian (62) represents a generalization of the static Skyrme-Faddeev-Niemi (SFN) theory $[37,38]$

$$
\mathcal{L}^{(\mathrm{SFN})}=\frac{1}{2 \lambda^{2}}\left(\partial_{i} \hat{n}^{a}\right)^{2}+\frac{e^{2}}{2}\left(\varepsilon^{a b c} \partial_{i} \hat{n}^{b} \partial_{j} \hat{n}^{c}\right)^{2} .
$$

In contrast to the SFN model, however, which was postulated on the basis of qualitative symmetry and renormal- ization group arguments [37,38], our Lagrangian (62) follows explicitly from the Yang-Mills dynamics and the Gaussian approximation to the vacuum wave functional. All coefficients are therefore fixed in terms of the IR scale $\mu$ and the coupling $g(\mu)$, i.e. Eq. (62) does not contain free parameters. While the $\hat{n}$ field of the SFN model is interpreted as a gauge-dependent local color direction in the vacuum, furthermore, our $\hat{n}$ field is manifestly gauge invariant. Besides allowing for a more direct physical interpretation, this has the additional advantage of preventing unwanted, colored Goldstone bosons from emerging when the $\mathrm{O}(3)$ symmetry of the Lagrangian (62) [or (23)] gets spontaneously broken.

Particular solutions of Eq. (61) are $\hat{n}^{a}=$ const, which belong to the class of translationally invariant vacua (cf. Sec. VIB), and $\hat{n}^{a}=\hat{x}^{a}$ (except at $r=0$ ) which is an example from the "hedgehog" solution family whose detailed discussion will be the subject of the following sections. The $\phi_{c 2}$ hedgehog has infinite action (since $U_{2 c, k}$ develops a monopole-type singularity at $\vec{x}=0$, cf. Sec. VII C) and its Lagrangian reduces to the FaddeevNiemi form (63). Equation (61) does probably also have cylindrically symmetric vortex solutions, i.e. analogs of the "baby Skyrmion" solutions [39] in models of Skyrme type.

The most interesting and many-faceted solution classes of the field equation (61), however, are expected to be knots, linked loops, and twists made of closed fluxtubes. Indeed, an intriguing variety of such topological soliton solutions was found numerically for the SFN part (63) of the Lagrangian (62) in Refs. [38,40]. These solutions generally lack axial symmetry and carry a finite Hopf charge ${ }^{12}$ $q_{H}^{(c 2)} \neq 0$. Moreover, their number and complexity increases strongly with the value of $\left|q_{H}^{(c 2)}\right|$. As in the higher- $|Q|$ solution sectors discussed previously, a practically useful saddle-point expansion thus requires an effective criterion for selecting the relevant contributions in a controlled fashion.

Such a criterion can be established by showing that the action $\Gamma^{\left(\phi_{c 2}\right)}$ based on the Lagrangian (62) is bounded from below by a monotonically increasing function of $\left|q_{H}\right|$. In fact, this just requires a straightforward adaptation of a known bound on the SFN action [41]: combining the obvious inequalities

$\Gamma\left[\phi_{c 2}, \hat{n}\right] \geq \frac{\mu}{g^{2}(\mu)} \int d^{3} x\left[\left(\partial_{i} \hat{n}^{a}\right)^{2}+\frac{1}{\mu^{2}}\left(\varepsilon^{a b c} \partial_{i} \hat{n}^{b} \partial_{j} \hat{n}^{c}\right)^{2}\right]$

\footnotetext{
${ }^{12}$ Despite the topological stability of solitons with nonvanishing $q_{H}$ in the submodel (62), it might be possible to "unwind" and thus destabilize them by excitations into the $\phi$ direction of the complete theory. This possibility deserves further investigation.
} 


$$
\geq \frac{2}{g^{2}(\mu)}\left[\int d^{3} x\left(\partial_{i} \hat{n}^{a}\right)^{2}\right]^{1 / 2}\left[\int d^{3} x\left(\varepsilon^{a b c} \hat{n}^{a} \partial_{i} \hat{n}^{b} \partial_{j} \hat{n}^{c}\right)^{2}\right]^{1 / 2}
$$

[the first one holds because the omitted term in the Lagrangian (62) is manifestly non-negative; the second one is a consequence of $(a-b)^{2} \geq 0$ for any real $\left.a, b\right]$ with the Sobolev-type inequality [41]

$$
\begin{aligned}
\left|q_{H}[\hat{n}]\right|^{3 / 2} \leq & \frac{1}{2^{13 / 2} 3^{3 / 4} \pi^{4}} \int d^{3} x \sqrt{\left(\varepsilon^{a b c} \hat{n}^{a} \partial_{i} \hat{n}^{b} \partial_{j} \hat{n}^{c}\right)^{2}} \\
& \times \int d^{3} x\left(\varepsilon^{a b c} \hat{n}^{a} \partial_{i} \hat{n}^{b} \partial_{j} \hat{n}^{c}\right)^{2}
\end{aligned}
$$

and a simple inequality due to Ward [42],

$$
\left[\left(\partial_{i} \hat{n}^{a}\right)^{2}\right]^{2} \geq 2\left(\varepsilon^{a b c} \hat{n}^{a} \partial_{i} \hat{n}^{b} \partial_{j} \hat{n}^{c}\right)^{2},
$$

yields the bound

$$
\Gamma\left[\phi_{c 2}, \hat{n}\right] \geq \frac{2^{9 / 2} 3^{3 / 8} \pi^{2}}{g^{2}(\mu)}\left|q_{H}[\hat{n}]\right|^{3 / 4} .
$$

$\left(2^{9 / 2} 3^{3 / 8} \pi^{2} \simeq 337.17\right)$ This bound is rather rough and could be made more stringent by incorporating the third term of the Lagrangian (62) and by refining the Sobolev inequality (66) which is expected to remain valid with about half of the factor on its right-hand side [42].

The probably most important consequence of the above results is the new dynamical framework and physical interpretation which they provide for Faddeev-Niemi-type knot solutions. In our approach, the knots represent specific gauge-invariant IR degrees of freedom whose underlying gluon field ensembles carry a collective Hopf charge. As a matter of fact, this may put the tentative identification of knot solutions with glueballs, as advocated in Refs. $[38,43]$ by generalizing the fluxtube picture for quark-antiquark mesons, on a gauge-invariant basis. (The original interpretation was somewhat obscured by the association of the $\hat{n}$ field with a local color direction [38].) In any case, the appearance of saddle-point solutions related to glueballs (in the $Q=0$ sector) would not be surprising since the latter are candidates for gluonic IR degrees of freedom in their own right. Moreover, in our framework the "glueball content" of the knot solutions (and of other IR fields) can be analyzed more directly, e.g. by investigating their contributions to the saddle-point expansion of glueball correlation functions. As a precursor and complement, one could furthermore calculate the higher-dimensional vacuum condensates which provide the principal input for the operator product expansion of glueball correlators. Contributions from individual IR degrees of freedom to the low-lying glueball spectrum could then be traced by duality sum rules [44].

Finally, it may be worth recalling that our framework for the knot dynamics makes the analysis of relations between the solutions of Eq. (61) and the underlying gluon fields possible. If links between the knots and relevant gauge field configurations with robust characteristics — such as topologically nontrivial pure-gauge fields in a nonlinear maximally Abelian gauge [45] or center vortices [6]exist, they might even be traceable by analytical methods. A fully quantitative survey of the knot solution sector, however, will require a devoted numerical effort ${ }^{13}$.

\section{Hedgehog solutions}

The existence of Skyrmions [29] in nonlinear $\sigma$-models with higher-derivative interactions suggests that our field equations (26) and (27) have topological soliton solutions of hedgehog type,

$$
\hat{n}^{a}(\vec{x})=\hat{x}^{a}, \quad \phi(\vec{x})=\phi^{(h h)}(r)
$$

$\left(\hat{x}^{a} \equiv \vec{x} / r, r \equiv|\vec{x}|\right)$, as well. Their SU(2) "grand spin" symmetry characterizes the invariance of the fields under simultaneous spatial and internal rotations and implies a substantial simplification of their dynamics. [For gauge groups $\mathrm{SU}(N)$ with $N>2$, the three components of $\hat{x}$ parametrize the part of the $\hat{n}^{a}$ field which is associated with the trivially embedded $S U(2)$ subgroup.] In the present section, we discuss general properties of the hedgehog fields and derive their Lagrangian and saddle-point equation. In Secs. VIE and VII, we find the most important solution classes explicitly and explain their physical interpretation.

The principal topological characteristic of the hedgehog configurations is their $\pi_{3}\left(S^{3}\right)$ winding number $Q$. For fields of the form (69), the integral representation (35) yields

$$
\begin{aligned}
Q\left[\phi^{(h h)}\right]= & \frac{1}{2 \pi} \int_{0}^{\infty} d r \phi^{(h h) \prime}\left(\cos \phi^{(h h)}-1\right) \\
= & \frac{1}{2 \pi}\left[\sin \phi^{(h h)}(\infty)-\sin \phi^{(h h)}(0)+\phi^{(h h)}(0)\right. \\
& \left.-\phi^{(h h)}(\infty)\right] .
\end{aligned}
$$

(Being a topological invariant, $Q$ depends only on the boundary values of the $\phi$ field.) As established in Sec. VB, finite-action fields carry integer values of $Q$, and Eq. (70) confirms this explicitly. Indeed, the parametrization (22) for hedgehog fields (69) implies that welldefined $U$ fields have $\phi^{(h h)}(0)=2 k_{1} \pi$ and that finiteaction fields must additionally satisfy $\phi^{(h h)}(\infty)=2 k_{2} \pi$ (see below) where $k_{1,2}$ and thus $Q=k_{1}-k_{2}$ are integers. Nevertheless, it is instructive to consider the more general boundary conditions

\footnotetext{
${ }^{13}$ Analytical solutions of knot equations of the type (61) are difficult to find in part because the obvious ansätze tend to fail. A few numerical knot solutions with small $\left|q_{H}\right|$ would probably be sufficient for the saddle-point expansion, however, since contributions to physical amplitudes are limited by the stringent action bound (68), especially when $Q=q_{H}=0$ solutions contribute simultaneously.
} 


$$
\begin{gathered}
\phi^{(h h)}(0)=n \pi, \quad \phi^{(h h)}(\infty)=m \pi, \\
Q\left[\phi^{(h h)}\right]=\frac{n-m}{2}
\end{gathered}
$$

( $n, m$ integer) which admit infinite-action fields with halfinteger winding numbers as well (for either $m$ or $n$ odd) ${ }^{14}$. We will show in Sec. VII that hedgehog solutions to the saddle-point Eqs. (26) and (27) under the boundary conditions (71), both with finite and infinite action, can indeed be found. Those with $Q= \pm 1$ will be of particular importance since they probably dominate all $Q \neq 0$ contributions to the saddle-point expansion. This is because of the bound (43) and because of Skyrme-model type arguments [29] which suggest that the minimal-action solutions in the $|Q|=1$ sectors are hedgehogs.

In addition, all hedgehog fields carry one unit of the monopole-type charge $q_{m} \in \pi_{2}\left(S^{2}\right)$. This becomes explicit when evaluating the ( $\phi$ independent) integral representation (36) for $q_{m}$ with $\hat{n}^{a}=\hat{x}^{a}$ :

$$
q_{m}^{(h h)}:=q_{m}[\hat{x}]=\frac{1}{4 \pi} \int d \sigma_{i} \frac{\hat{x}_{i}}{r^{2}}=1 .
$$

Obviously, $q_{m}^{(h h)}$ is independent of the boundary conditions for $\phi$ and therefore of $Q$. The field with $q_{m}^{(h h)}=-1$ (the "antimonopole") is obtained by changing the sign of $\hat{x}_{i}$, which corresponds to $U \rightarrow U^{\dagger}$ for fixed $\phi$. Equation (37) reveals, finally, that the Hopf charge of all hedgehog configurations vanishes.

The dynamics of the hedgehog fields is governed by the soft-mode Lagrangian. Since $\hat{x}$ is an identical solution of the general field equation (27) for $\hat{n}$, Eq. (23) can be directly specialized to $\hat{n}^{a}=\hat{x}^{a}$. Hence the integration over angles becomes trivial and the hedgehog action turns into

$$
\Gamma\left[\phi^{(h h)}, \hat{x}\right]=\int_{0}^{\infty} d r \mathcal{L}^{(h h)}(r)
$$

where $\mathcal{L}^{(h h)}$ is a $\phi^{(h h)}$-dependent radial Lagrangian. After substituting the ansatz (69) into the full Lagrangian (23), dropping total derivatives, suppressing the superscript of $\phi^{(h h)}$ and again denoting radial derivatives $d / d r$ by a prime, one arrives at

$$
\begin{aligned}
\mathcal{L}^{(h h)}(r)= & \frac{\pi}{g^{2}(\mu) \mu}\left[\frac{1}{2}\left(r \phi^{\prime \prime}\right)^{2}+\left(3+\mu^{2} r^{2}\right)\left(\phi^{\prime}\right)^{2}\right. \\
& \left.+4 \mu^{2}(1-\cos \phi)\right] .
\end{aligned}
$$

All terms in $\mathcal{L}^{(h h)}$ are non-negative. As a consequence, each of them has to vanish individually at any absolute action minimum. The complete set of hedgehog vacua is

\footnotetext{
${ }^{14}$ The half-integer winding numbers cannot be associated with the degree of a map since the latter is defined for maps between compact manifolds only (which, in our context, requires finite action).
}

therefore $\left(\phi_{c, k}^{(h h)}, \hat{n}^{(h h)}\right)=(2 k \pi, \hat{x})$ and forms a subset of the translationally invariant center elements (48). Hence, any finite-action hedgehog solution has to approach one of these constant minima when $r \rightarrow \infty$. The constant solutions $\phi_{c 2, k}^{(h h)}=(2 k+1) \pi$, on the other hand, are maxima of the action. A representative of this type was already encountered in Sec. VIC.

The radial equation for $\phi^{(h h)}$ can be derived by inserting the ansatz (69) into the general field equation (26) or by varying the radial Lagrangian (74). Either way, the result is

$$
\begin{aligned}
r^{2} \phi^{\prime \prime \prime \prime}+4 r \phi^{\prime \prime \prime}-2\left(2+\mu^{2} r^{2}\right) \phi^{\prime \prime} & -4 \mu^{2} r \phi^{\prime} \\
& +4 \mu^{2} \sin \phi=0,
\end{aligned}
$$

i.e. an ordinary nonlinear differential equation of fourth order and of Fuchsian type. The associated boundary value problem can be solved numerically with moderate computer resources. The exploration of the full solution space is facilitated by two discrete symmetries of Eq. (75) which imply that any solution $\bar{\phi}(r)$ gives rise to the additional solutions $-\bar{\phi}(r)$ and $\bar{\phi}(r)+2 n \pi$. The former is a consequence of $\Gamma[U]=\Gamma\left[U^{\dagger}\right]$ while the latter reflects periodicity in the angular variable $\phi$.

Not surprisingly, the field equation (75) comprises the Gribov equation [46] in the terms proportional to $\mu^{2}$. Those terms originate from the nonlinear- $\sigma$-model part of the Lagrangian (74) and, due to the bound (12), dominate

the higher-derivative terms in the validity range of the soft gradient expansion (16). [Nevertheless, the $\mu$-independent terms of Eq. (75) play a crucial role in the transition region between the boundary values of $\phi$ where the impact of the additional derivatives makes stable soliton solutions possible.] The analogy between the nonlinear potential term $\propto \sin \phi$ and a one-dimensional pendulum in a gravitational field $^{15}$, often employed to characterize the solution spectrum of the Gribov equation, therefore applies to Eq. (75) as well. The stable (unstable) equilibrium positions of the pendulum correspond to $\phi=\pi(\phi=0)$, modulo a multiple of $2 \pi$ which represents additional full turns. We will show in Sec. VII that this analogy is able to describe the qualitative behavior of all numerical solutions.

\section{E. Analytical hedgehog solutions by series expansion}

The $(\phi, \hat{n})$ parametrization of the $U$ field implies that regular solutions $\phi^{(h h)}$ of the radial hedgehog Eq. (75) must approach a multiple of $2 \pi$ at the origin. Hence their small- $r$ behavior can be determined analytically, either by expanding the nonlinearity of Eq. (75) into powers of a small deviation $\delta \phi(r)$ from the constant action minima $\phi_{c, k}^{(h h)}=2 k \pi$ or by expanding the $r$ dependence of the full

\footnotetext{
${ }^{15}$ This analogy becomes more explicit after replacing $r$ by the logarithmic variable $t=\ln \left(r / r_{0}\right)$.
} 
solution into a Frobenius series. Similarly, finite-action solutions can be obtained for $r \rightarrow \infty$ by asymptotically expanding around the hedgehog vacua. Inside their regions of validity, those expansions provide useful insights into the qualitative behavior of hedgehog solutions as well as quantitative checks on their numerical solutions.

We start by deriving the solutions of the linearized hedgehog equation and the corresponding power series expansion around the origin. Inserting the ansatz

$$
\phi(r)=2 k \pi+\delta \phi(r)+O\left(\delta \phi^{2}\right)
$$

into the radial saddle-point equation (75) and retaining only terms up to first order in $\delta \phi$, one arrives at the fourth-order linear differential equation

$$
\begin{aligned}
r^{2} \delta \phi^{\prime \prime \prime \prime}+4 r \delta \phi^{\prime \prime \prime}-2\left(2+\mu^{2} r^{2}\right) \delta \phi^{\prime \prime} & -4 \mu^{2} r \delta \phi^{\prime} \\
& +4 \mu^{2} \delta \phi=0
\end{aligned}
$$

which can be solved analytically by standard techniques. The general solution is a superposition of four linearly independent base solutions $\delta \phi_{i}$,

$$
\delta \phi(r)=\sum_{i=1}^{4} \tilde{c}_{i} \delta \phi_{i}(r)
$$

whose real, dimensionless coefficients $\tilde{c}_{i}$ have to be specified by imposing initial or boundary conditions. The base solutions $\left\{\delta \phi_{i}\right\}$ are

$$
\begin{aligned}
& \delta \phi_{1}=\mu r, \quad \delta \phi_{2}=\frac{1}{\mu^{2} r^{2}}, \\
& \delta \phi_{3}=(1+\sqrt{2} \mu r) \frac{e^{-\sqrt{2} \mu r}}{\mu^{2} r^{2}}, \\
& \delta \phi_{4}=(1-\sqrt{2} \mu r) \frac{e^{+\sqrt{2} \mu r}}{\mu^{2} r^{2}} .
\end{aligned}
$$

The requirements of regularity and uniqueness dictate two of the boundary conditions. The first one, $\delta \phi(0)=0$, implies $\phi(0)=2 k \pi$ and thus ensures uniqueness at $r=$ 0 while the second one, $\delta \phi^{\prime \prime}(0)=0$, is subsequently imposed by the behavior of the base solutions (79). As a consequence, the small- $r$ behavior of the regular solutions gets restricted to

$$
\phi(r) \stackrel{r \rightarrow 0}{\longrightarrow} 2 n \pi+c_{1} \mu r+c_{2} \mu^{3} r^{3}+O\left(\mu^{4} r^{4}\right),
$$

where the constants $c_{1,2}$ are linear combinations of the $\tilde{c}_{1,3,4}$ whose values can e.g. be fixed by providing initial data for $\phi^{\prime}(0)$ and $\phi^{\prime \prime \prime}(0)$. All higher-order coefficients of the expansion are then determined.

Alternatively, one can obtain the solutions of the full, nonlinear saddle-point Eq. (75) towards $r \rightarrow 0$ by analytical continuation into a Frobenius series. A straightforward calculation yields

$$
\begin{aligned}
\phi(r)= & 2 n \pi+\phi_{1} r+\phi_{3} r^{3}+\frac{\mu^{2}}{14}\left(\phi_{3}+\frac{\phi_{1}^{3}}{30}\right) r^{5} \\
& +\frac{\mu^{2}}{2 \cdot 3^{3} 7}\left[\mu^{2}\left(\phi_{3}+\frac{\phi_{1}^{3}}{30}\right)+\frac{1}{2} \phi_{1}^{2} \phi_{3}-\frac{1}{5 !} \phi_{1}^{5}\right] r^{7} \\
& +O\left(r^{9}\right),
\end{aligned}
$$

where the coefficients $\phi_{1}$ and $\phi_{3}$ are again left to be determined by boundary conditions. Even-order derivatives of $\phi$ (or equivalently the coefficients $\phi_{2 k}$ ) vanish at $r=0$ while those of odd order, $\phi_{2 k+1}$, can be expressed in terms of $\phi_{1}$ and $\phi_{3}$. A comparison between Eqs. (78) and (81) shows that the solutions of the exact radial equation start to differ from those of the linearized equation at $O\left(r^{3}\right)$. Hence the series solution (81) permits a more accurate check of the numerical solutions over a larger radial interval.

Analogous expansions around the constant action minima $\phi_{c, k}^{(h h)}=2 k \pi$ hold asymptotically, i.e. towards $r \rightarrow \infty$, for all finite-action solutions. Infinite-action solutions of Eq. (75), finally, can be linearized around the constant solutions $\phi_{2, k}^{(h h)}=(2 k+1) \pi$ which they approach at one or both ends of the radial domain. The resulting equation for $\delta \phi$ differs from Eq. (77) only in the sign of the $\delta \phi$ term. Its solutions are linear combinations of generalized hypergeometric functions.

\section{NUMERICAL ANALYSIS AND PHYSICAL INTERPRETATION}

We now turn to the numerical solution of the saddlepoint equation (75). Because of the periodicity in $\phi$, the range of boundary values can be limited without loss of generality to $\phi(0) \in] 0,2 \pi]$. Then regularity at the origin specifies $\phi(0)=2 \pi$ and imposes $\phi^{\prime \prime}(0)=0$ (see Sec. VIE). The value of the topological charge $Q$ fixes a third boundary condition,

$$
\phi(\infty)=2 \pi(1-Q),
$$

owing to Eq. (70). Hence all regular hedgehog solutions in a given $Q$-sector can be found by just varying the value of a fourth boundary condition. In the following, we will use the initial slope $\beta:=\phi^{\prime}(0)$ for this purpose. At the end of the section, we will also search for irregular solutions with $\phi(0)=\pi$.

\section{A. Instanton classes}

We begin our exploration of the hedgehog solution space by searching for regular finite-action solutions which, as established in Sec. VB, carry integer values of $Q$. The numerical analysis shows (and the pendulum analogy in Sec. VIIC will explain) that only one solution of this type exists in each $Q$ sector. In the simplest case, $Q=0$, this is just the translationally invariant vacuum solution $\phi^{(h h)}=$ $2 \pi$. 
For $Q=1$ we obtain the prototypical nontrivial hedgehog solution, depicted in Fig. 2. In order to clarify its physical interpretation, we first note that it shares the $\pi_{3}\left(S^{3}\right)$ homotopy classification, encoded in the topological charge $Q[U]$ of the relative gauge orientation $U=$ $U_{-}^{-1} U_{+}$, with the Yang-Mills instanton [47]. Of course, both also share the saddle-point property, as the instanton minimizes the classical Euclidean Yang-Mills action in the $Q=1$ sector. In order to trace the association with a YangMills instanton of size $\rho$ further, we inspect the corresponding relative gauge orientation $U_{I, \mathrm{YM}}$. It is of hedgehog type as well, and its $\vec{x}$-dependence in (Euclidean) temporal gauge is known to be [48]

$$
\phi_{I, \mathrm{YM}}(r)=-\frac{2 \pi r}{\sqrt{r^{2}+\rho^{2}}} .
$$

For comparison with our $Q=1$ solution, we have included $\phi_{I, \mathrm{YM}}$ with $\rho=2 \mu^{-1}$ as the dashed curve in Fig. 2 (and adapted it to our periodicity interval convention by adding $2 \pi$ ). Both solutions are surprisingly similar. This implies that the dominant contributions from all $Q=1$ gauge field orbits to the vacuum overlap have a relative gauge orientation close to that of the Yang-Mills instanton and indicates that the $Q=1$ hedgehog solution primarily summarizes contributions from the instanton orbit. (Of course, one would not expect exact agreement since our solutions contain scale-symmetry breaking quantum corrections ${ }^{16}$ and contributions from other $Q=1$ gauge fields as well.) Generalizing the above findings to multi-instantons, we will therefore refer to the unique regular finite-action solution of Eq. (75) with integer $Q$ as the " $Q$-instanton class."

Our hedgehog saddle-point equation (75) and its instanton-class solutions derive from the gradientexpanded soft-mode Lagrangian (16). It is instructive to compare the resulting one-instanton effects with a variational estimate of one-instanton contributions to the bare action (5) in Ref. [49]. By approximately minimizing the bare action with one-parameter families of trial functions similar to the instanton profile (83) and using qualitative scaling arguments, it was suggested in Ref. [49] that radiative corrections can restrict the instanton size. Our explicit saddle-point solutions confirm this expectation and make the dynamical size stabilization manifest. We have already traced the underlying mechanism to the virial theorem (32) which is independent of details of the softmode dynamics and thus overcomes the chronic infrared instability of dilute instanton gases [50] in a rather generic way. For $\mu \simeq 1.5 \mathrm{GeV}$, the size $\rho \simeq 2 \mu^{-1}$ of the 1 -instanton-class solution agrees inside errors with the results of

\footnotetext{
${ }^{16}$ Recall that the analogous scale-symmetry breaking due to one-loop corrections in Yang-Mills theory is unable to stabilize the instanton size distribution.
}

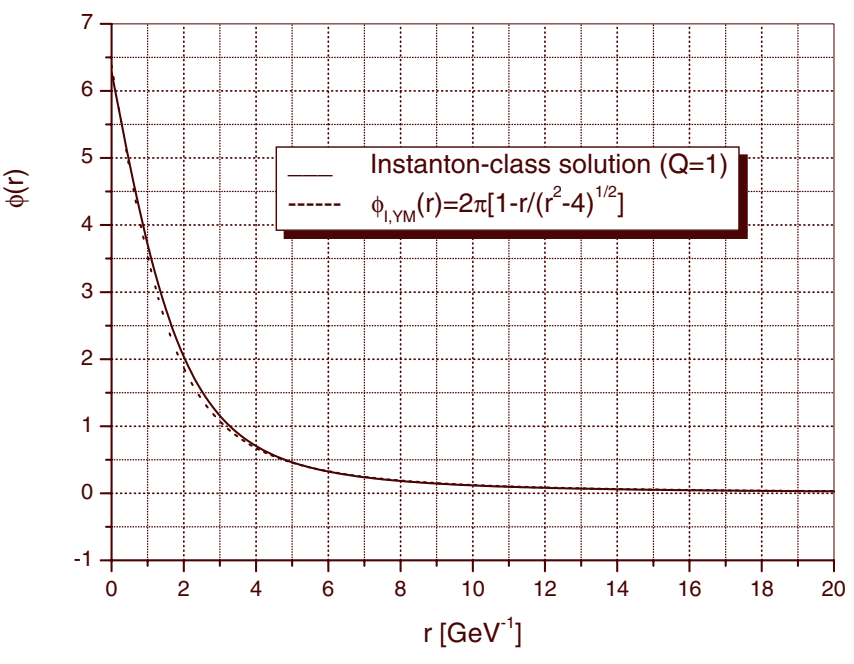

FIG. 2 (color online). The canonical 1-instanton-class solution with $Q=1$.

instanton liquid model [52] and lattice [53] simulations. ${ }^{17}$ Moreover, it confirms that the two leading terms of the gradient expansion (13) yield a sufficiently accurate approximation to the instanton action [cf. the comments below Eq. (17)]

Our 1-instanton-class profile function $\phi_{I}(r)$ is similar to the one found by approximately minimizing the bare action (5) variationally with a one-parameter set of trial functions [49]. This suggests that the bulk of the instanton dynamics and size distribution is generated by soft modes, as one would intuitively expect, and that our approach therefore provides an efficient framework for the treatment of these and other vacuum fields. In contrast to variational approaches, furthermore, it allows to systematically account for the contributions of all saddle points (most of which are not of hedgehog form). In fact, already in the hedgehog sector we will find solutions with unprecedented shapes, more complex than Eq. (83). With little guidance for the choice of suitable trial functions, such solutions would be difficult to find variationally.

According to Eq. (71), all monotonic hedgehog solutions with $Q>0(Q<0)$ have negative (positive) slopes $\beta=\phi^{\prime}(0)$ at the origin. The anti-instanton class with $Q=$ -1 , for example, is characterized by the instanton boundary value with opposite sign, $\beta_{\bar{I}}=-\beta_{I}$, and can be obtained without further calculation: it results from the combined symmetry transformations $\phi \rightarrow-\phi$ and $\phi \rightarrow$ $\phi+4 \pi$ on the instanton-class solution. Hence the $Q=$ \pm 1 instanton classes have degenerate action values, precisely as their Yang-Mills counterparts.

Multi-instanton-class solutions are characterized by integer topological charges $Q \geq 2$. The modulus $\left|\beta_{Q, I}\right|$ of

\footnotetext{
${ }^{17}$ The dynamical mass scale $\mu$ and the average instanton size might be related to an effective IR fixed point of the strong coupling [51].
} 
their (negative) initial slope grows monotonically with $Q$, i.e.

$$
\left|\beta_{I, Q^{\prime}}\right|>\left|\beta_{I, Q}\right| \text { for } Q^{\prime}>Q \text {. }
$$

As in the 1-instanton case, the action-degenerate multianti-instanton classes with $Q \leq-2$ can be constructed by flipping the sign of the corresponding multi-instanton classes and by adding $4 \pi$. Hence the relation between the inital slopes of the (anti)instanton solutions generalizes to all $Q$ :

$$
\beta_{\bar{I},-Q}=-\beta_{I, Q}
$$

Remarkably, the $|Q| \geq 2$ hedgehog solutions correspond to special arrangements of the underlying Yang-Mills multi-(anti)instantons. In fact, the relative gauge orientation $U=U_{-}^{-1} U_{+}$of a multi-instanton configuration is of hedgehog type only if all individual (anti)instantons are centered at the origin. This raises the question whether $|Q| \geq 2$ Yang-Mills instantons with separated individual positions are at least approximately represented by other solutions of the saddle-point equations (26) and (27). Experience from Skyrme-type models, whose analogous $|Q| \geq 2$ solutions are well approximated by rational [54] or harmonic [55] maps, suggests that similar types of nonhedgehog field configurations approximate higher- $Q$ solutions of Eqs. (26) and (27) as well.

The action of the 1-instanton-class solution is large $\left(\Gamma_{I} \sim 220 / g^{2}\right.$ at $\left.\mu=1 \mathrm{GeV}\right)$, as the large action of typical Yang-Mills instantons, and its direct impact on the saddlepoint expansion is therefore small ${ }^{18}$. Moreover, the action bound (43) implies that higher- $Q$ instanton classes should be irrelevant for most amplitudes, with potential exceptions as mentioned in Sec. V C. Instanton "liquid" vacuum models (ILMs) [5] suggest that in Yang-Mills theory the physically important contributions originate instead from ensembles of instantons and anti-instantons with equal average densities. It remains to be checked whether analogous contributions are approximately represented by nontrivial $Q=0$ solutions of Eqs. (26) and (27). If such solutions exist, our above results imply that they cannot be of hedgehog type.

\section{B. Meron classes}

We now extend our search to hedgehog solutions with infinite action. Although their relevance for the saddlepoint expansion is not obvious, one may speculate that their infinite-action suppression could be overcome by

\footnotetext{
${ }^{18}$ Note that the 1 -instanton class does not saturate the action bound (43) and hence does not solve the Bogomol'nyi-type Eq. (44). This is in constrast to the Yang-Mills instanton which is the absolute minimum of the Euclidean Yang-Mills action in the $Q=1$ sector and therefore self-dual.
}

additional mechanisms (see below). Our chief motivation for discussing them here, however, derives from their association with the infinite-action meron solutions [7] of the classical (Euclidean) Yang-Mills equation.

Hedgehog solutions with infinite action are far more the rule than the exception. In fact, all regular $(\phi(0)=2 \pi)$ solutions of Eq. (75) with initial slopes $\beta$ between the discrete set of instanton-class values $\beta_{I, Q}$ have infinite action since they approach $\phi_{M}(\infty)=(2 k+1) \pi$ towards spacial infinity. The latter furthermore implies that the asymptotic fields $U_{M}(|\vec{x}| \rightarrow \infty)$ remain angle-dependent. Exactly the same behavior characterizes the relative gauge orientations $U=U_{-}^{-1} U_{+}$of Yang-Mills merons in temporal gauge, which are of hedgehog type as well. Furthermore, Eq. (82) shows that solutions with $\phi(\infty)=$ $(2 k+1) \pi$ carry half-integer topological charge $Q$, again as the Yang-Mills merons. In analogy with the nomenclature of the previous section, we will therefore refer to these solutions as " $2 Q$-meron classes."

The profile function $\phi_{M}(r)$ of a typical 1-meron-class solution with $Q=1 / 2$ is drawn in Fig. 3. A direct comparison with the profile of the Yang-Mills meron in temporal gauge,

$$
\phi_{M, \mathrm{YM}}(r)=\frac{1}{2} \lim _{\rho \rightarrow 0} \phi_{I, \mathrm{YM}}(r)=-\pi \theta(r)
$$

(where $\theta$ is the step function), is complicated by the fact that the classical meron is pointlike while our solutions incorporate quantum effects which break dilatational symmetry and stabilize their size at a finite value. Such effects, which also cause our solution $\phi_{M}(r)$ to become nonmonotonic by overshooting in the transition region, are expected to smoothen the singularity of the Yang-Mills meron as well. For easier comparison, we therefore draw the YangMills meron profile in Fig. 3 (dashed curve) with the finite

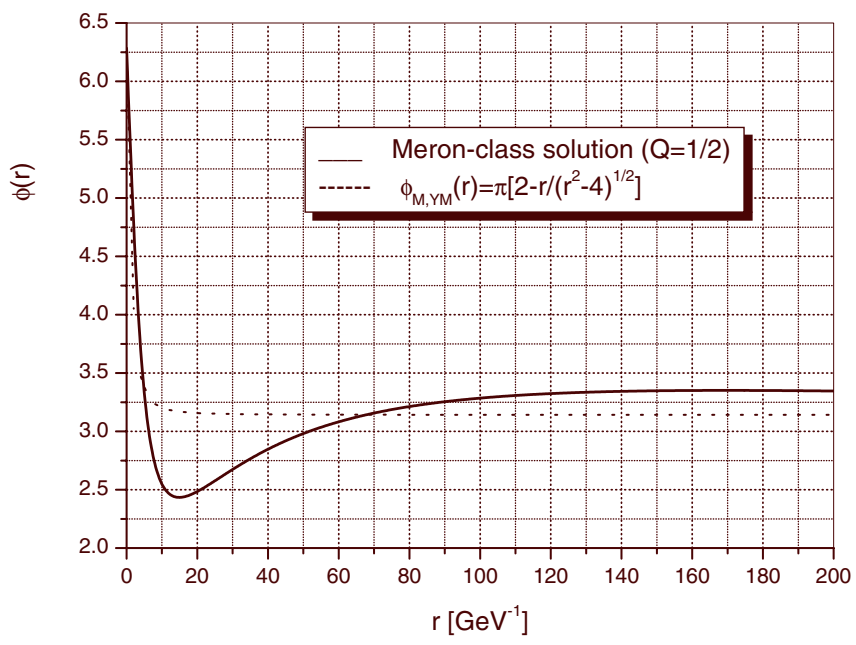

FIG. 3 (color online). A typical meron-class solution with $Q=$ $1 / 2$. 
size $\rho=2 \mu$ of the instanton-class solutions (and adapt it to our periodicity interval convention).

Our multimeron classes with $|Q|>1 / 2$ include only hedgehog fields with half-integer topological charge because solutions with even "meron number" and correspondingly integer $Q$ coincide with the $Q$-instanton classes. This is expected since the relative gauge orientation of Yang-Mills multimerons is of hedgehog type only if all individual merons sit on top of each other. Such configurations, when carrying integer overall values of $Q$, coalesce into the corresponding Yang-Mills instantons and in our framework those are represented by the $Q$ instanton-class solutions.

While the behavior of the multi-meron-class solutions with $Q \geq 3 / 2$ is qualitatively rather similar to that of the 1 meron class, the size and strength scales may differ substantially. This is exemplified in Fig. 4 which shows a typical 3-meron class solution. More generally, all solutions with half-integer $Q$ come in families of continuously varying sizes. As already alluded to, the reason is that their size depends on a second mass scale $\beta_{M} \equiv \phi_{M}^{\prime}(0)$ (in addition to $\mu$ ) and that solutions for all values of $\beta_{M}$ in the finite intervals

$$
\beta_{I, Q}>\beta_{M, Q+1 / 2}>\beta_{I, Q+1}
$$

(where $Q \geq 0$, i.e. $\beta \leq 0$, and $\beta_{I, Q=0}=0$ are implied) can be found. As in the instanton sector, multi-anti-meron classes with negative $Q$ are obtained from the positive- $Q$ solutions by changing their sign and adding $4 \pi$.

The variable mass scales $\beta_{M, Q}$ in the meron sectors imply that there are infinitely more meron than instantonclass solutions. Hence it becomes tempting to speculate that the meron entropy contributions to the weight function in functional integrals over $U$ may be able to overcome the

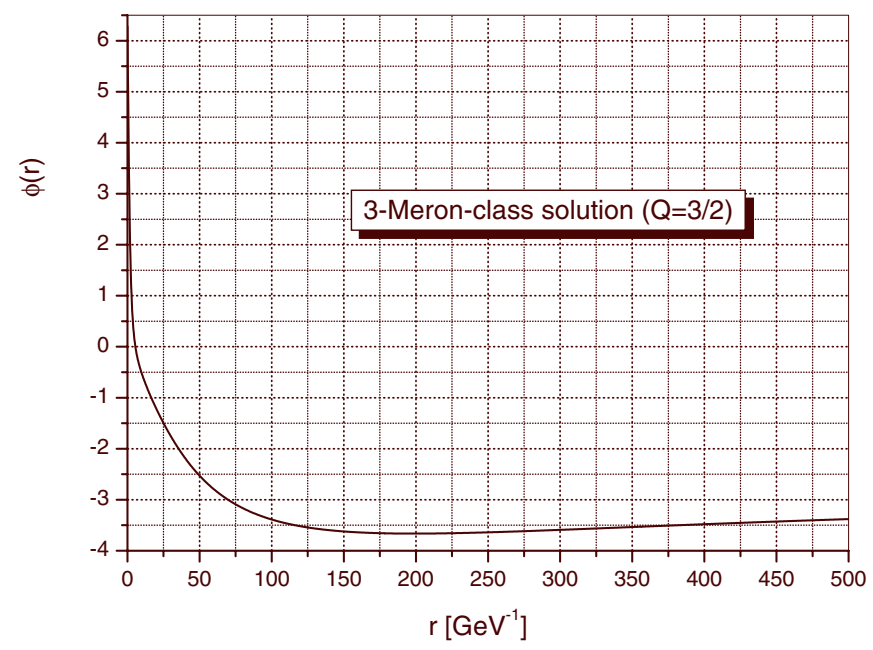

FIG. 4 (color online). A 3-meron-class solution with $Q=3 / 2$. infinite-action suppression. If so, this mechanism would shed new light on the physical interpretation not only of our solutions but probably of the Yang-Mills merons as well (whose potential physical role remains controversial). Furthermore, it might suggest a more efficient saddle-point expansion in which the free energy is extremized. These issues deserve further investigation.

\section{Singular hedgehog solutions}

Above we have classified all regular hedgehog solutions, i.e. those which satisfy the initial condition $\phi(0)=2 \pi$. Now we are going to examine the remaining solution classes of the radial field equation (75). Its members share the boundary condition $\phi(0)=\pi$, display a rather complex spacial structure and are characterized by a monopoletype singularity at the origin (i.e. they solve the field equation everywhere except at $\vec{x}=0$ ).

In order to understand the qualitative behavior of both regular and irregular hedgehog solution classes from a common perspective, it is useful to elaborate on the analogy between the hedgehog equation (75) and the pendulum equation which was mentioned in Sec. VID. According to this analogy, the instanton classes correspond to exactly $Q$ full turns of the pendulum, with the sign of $Q$ indicating the direction of the rotation. The pendulum mass starts in the unstable equilibrium position at time $t=\ln r=-\infty$ with just enough initial speed $\beta=\phi^{\prime}(0)$ to finally end up there again at $t=\ln r=+\infty$. This analogy implies, in particular, that there is exactly one regular hedgehog solution for each integer $Q$ and that the constant $\phi=2 \pi$ is the only regular solution with $Q=0$. The meron-class solutions start from the unstable equilibrium position as well. However, their initial velocity $\beta$ is insufficient for completing all turns in full. The last one remains unfinished, i.e. the pendulum swings back, oscillates around and finally settles into the stable equilibrium position. Hence all meron-class solutions have half-integer $Q$ and are nonmonotonic.

For the irregular solutions, on the other hand, the pendulum starts at the stable equilibrium position $\phi=\pi$. When not provided with sufficient initial speed $\beta$ to complete a full turn, it just performs damped oscillations around $\phi=\pi$. The corresponding solution has $Q=0$ and is depicted in Fig. 5. (Nontrivial hedgehog solutions with $Q=0$ are necessarily irregular at the origin.) When $|\beta|$ is sufficiently large, however, the pendulum can perform $Q$ full turns before settling into the stable equilibrium position. As a consequence, all these solutions have integer $Q \neq 0$ and infinite action [cf. Eq. (74)]. Pursuing the analogy further, one would also expect irregular solutions with initial speed $\beta$ exactly as needed to end up at the unstable equilibrium position when $r \rightarrow \infty$ (after possibly completing a number of full turns). Such configurations would carry a half-integer topologically charge $Q$ and a finite action. For obvious reasons they turn out to be highly 


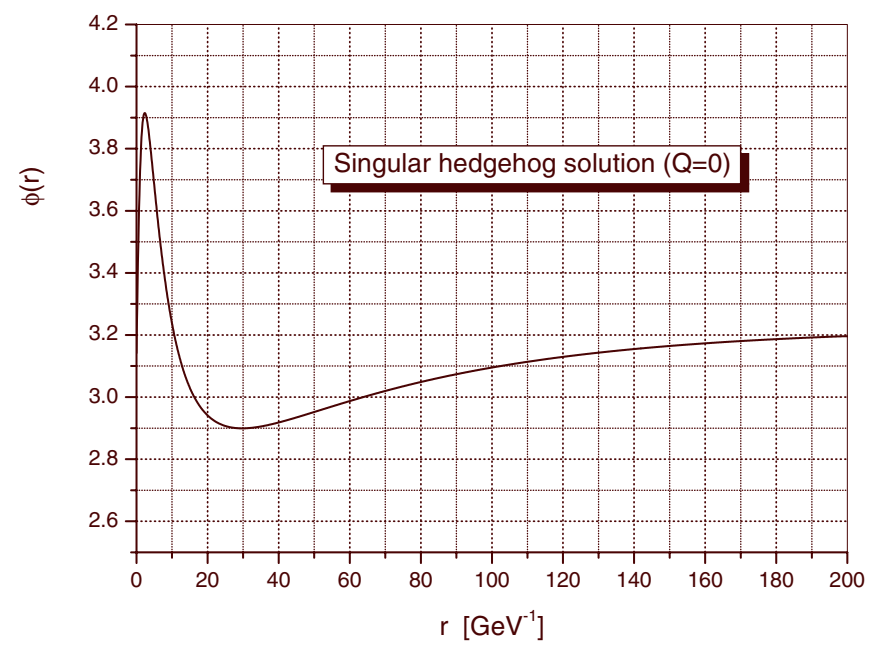

FIG. 5 (color online). An example for a nontrivial $Q=0$ hedgehog solution with monopole-type behavior at the origin.

sensitive to variations of the boundary condition, however, and therefore difficult to establish numerically.

In contrast to the instanton and meron classes, the singular hedgehog solutions with integer $Q$ (half-integer- $Q$ ) carry infinite (finite) action and do not seem to have obvious analogs among the solutions of the classical Yang-Mills equation. Their interpretation and their relevance for the saddle-point expansion remains to be clarified. A potential physical impact of such solutions seems to require mechanisms which could both smoothen their singularity and overcome their infinite-action suppression.

\section{SUMMARY AND CONCLUSIONS}

We have constructed an operative saddle-point expansion for the Yang-Mills vacuum overlap amplitude in terms of gauge-invariant soft-mode fields and used it to identify a wide range of new gluonic IR degrees of freedom. The latter emerge as the saddle points of an effective action which gather specific contributions from dominant gauge field families to low-energy amplitudes and are both gauge invariant and universal (i.e. their definition makes no reference to particular amplitudes). On the basis of a gaugeprojected Gaussian approximation for the vacuum wave functional the IR degrees of freedom can be derived explicitly. Besides providing the principal input for a systematic saddle-point expansion of soft amplitudes, they turn out to shed new light on the organization of the low-energy Yang-Mills dynamics.

Our survey of the saddle-point space uncovered a diverse spectrum of IR degrees of freedom which inherit a variety of topological charges from the gauge group and obey associated Bogomol'nyi-type action bounds. The scale stability of the solutions, indispensable for a viable saddle-point expansion, is guaranteed by a virial theorem. Solutions with a relatively high degree of symmetry can be found either analytically or with moderate numerical effort. Since more symmetric solutions generally carry less action and hence play more important roles in the saddlepoint expansion, we have mainly focused on the investigation of their properties. Besides translationally invariant vacua and analytical solutions with a fixed relative gauge orientation, we found topological solitons of hedgehog, (vortex) link and knot types.

Some of the IR degrees of freedom have a robust and transparent physical interpretation in terms of the underlying gluon fields. The contributions from gauge orbits of Yang-Mills instanton and merons, in particular, are gathered by saddle points of hedgehog type (with equivalent, integer or half-integer topological charges) and represent vacuum tunneling in the Hamiltonian formulation of nonAbelian gauge theory. Although our hedgehog solutions include quantum fluctuations and potentially relevant contributions from other gluon fields, those in the instanton class turn out to approximately reproduce the relative gauge orientation between the in- and out-vacua of the Yang-Mills instanton. Quantum effects play a much more important role in the meron solution classes, on the other hand, whose finite extent they generate by smoothening the singularities of the classical Yang-Mills merons. (Nevertheless, their action still diverges.)

Among those IR fields with unit topological (instanton) charge, the single (anti-)instanton classes are expected to attain the absolute action minimum. As a consequence of the action bound, they should therefore dominate the saddle-point expansion in all nontrivial topological charge sectors. The size of the (anti-)instanton classes is dynamically stabilized. It turns out to be fixed at about twice the inverse IR gluon mass scale and agrees inside errors with instanton liquid model and lattice results. Hence the underlying virial theorem and the soft gluon mass generation conspire to overcome the chronic infrared diseases which plague dilute Yang-Mills instanton ensembles.

The sizes of the meron-class solutions with half-integer topological charge are of a more complex origin. Besides the dynamical gluon mass they depend on a second, variable mass scale which is encoded in a boundary condition. Hence meron classes exist in continuous size ranges and form a far bigger solution family than the instanton classes. This opens up the hypothetical possibility for their entropy to overcome the infinite-action suppression in functional integrals. Such a mechanism would not only help to clarify the physical impact of our solutions but also shed new light on the still controversial role of Yang-Mills merons themselves. In addition, there exists a third class of hedgehog solutions which contains a monopole-type singularity at the origin. These irregular solutions carry half-integer and integer (including zero) topological charges as well as mostly infinite action.

Several remarkable families of IR degrees of freedom turn out to be represented by topological solitons of 
Faddeev-Niemi type, i.e. by (potentially twisted) links and knots. In fact, our saddle-point dynamics contains a specific generalization of the Faddeev-Niemi Lagrangian and shows how it is embedded in the Gaussian approximation to the Yang-Mills vacuum wave functional. This puts Faddeev-Niemi theory into a new perspective, as the effective dynamics of dominant gauge field orbits with a collective Hopf charge, and provides the underlying unitvector field with a manifestly gauge-invariant meaning. The latter would strengthen a previous, tentative identification of knot solutions with glueballs. In our framework, such interpretations can be tested for example by evaluating the impact of the knot saddle points on suitable amplitudes (like glueball correlation functions).

More generally, our approach allows the evaluation of contributions from all relevant IR degrees of freedom to functional integrals of any soft amplitude by means of the saddle-point expansion. First calculations of this type, focusing on fundamental vacuum properties including gluon condensates and the topological susceptibility, are in progress. In addition, the gauge field content of any individual IR variable can be analyzed by applying standard functional techniques to the gluon-field integrals with which they are associated. Besides deepening the insight into the physical role of specific IR degrees of freedom, analyses of this type may also shed new light on the dynamical processes by which soft gauge fields organize themselves into collective degrees of freedom.

The diverse topological properties of the IR variables show that the gauge-projected Gaussian wave functional not only captures the homotopy structure of the gauge group but also sustains induced topologies (like the Hopf charge) which further characterize the saddle-point families. Owing to the typical robustness of topological properties, the related results should remain at least qualitatively valid beyond the Gaussian approximation. In addition, the saddle-point expansion engenders the means to map out limitations of the underlying vacuum wave functional systematically, e.g. by comparing suitable amplitudes with lattice data. Extensions to supersymmetric gauge theories would provide further (analytical) testing grounds, for example by tracing vestiges of the monopolebased confinement mechanism back to the vacuum functional. Such analyses could also guide the development of improved soft-mode actions beyond the Gaussian approximation, perhaps along the lines of those which are emerging in $2+1$ dimensional Yang-Mills theory, and generate systematic corrections to the IR variables.

Our approach opens up a variety of directions for future work. Among them are a more exhaustive survey of the saddle-point solution space, which should encompass potentially relevant approximate solutions, and the evaluation of vacuum amplitudes. The extension to full QCD requires the adaptation to the gauge group $\mathrm{SU}(3)$ and the implementation of quarks into the Gaussian wave functional, both of which are at least conceptually straightforward. Most topological properties, in particular, reside in the trivially embedded SU(2) color subgroup and will remain unaffected. A sufficiently complete treatment of the quarkgluon interactions and their impact on the effective action, however, appears more challenging. It would also be interesting to explore relations between our gauge-invariant IR degrees of freedom and gauge-dependent gluonic structures (e.g. monopoles, vortices, branes as well as certain Green functions) which play important roles in gauge-fixed formulations.

\section{ACKNOWLEDGMENTS}

Financial support from the Conselho Nacional de Desenvolvimento Científico e Tecnológico (CNPq) of Brazil as well as the hospitality and a visiting scientist grant at the $\mathrm{ECT}^{*}$ in Trento (Italy) are gratefully acknowledged.
[1] T. H. Hansson, K. Johnson, and C. Peterson, Phys. Rev. D 26, 2069 (1982).

[2] H. G. Dosch, Phys. Lett. B 190, 177 (1987); H. G. Dosch and Yu. A. Simonov, Phys. Lett. B 205, 339 (1988).

[3] H. B. Nielsen and P. Olesen, Nucl. Phys. B160, 380 (1979).

[4] See e.g. A. C. Kalloniatis and S. N. Nedelko, Phys. Rev. D 64, 114025 (2001).

[5] T. Schaefer and E. V. Shuryak, Rev. Mod. Phys. 70, 323 (1998); D. I. Diakonov, Prog. Part. Nucl. Phys. 51, 173 (2003); For an elementary introduction to instantons see H. Forkel, hep-ph/0009136.
[6] G. 't Hooft, Nucl. Phys. B138, 1 (1978); J. Greensite, Prog. Part. Nucl. Phys. 51, 1 (2003).

[7] V. de Alfaro, S. Fubini, and G. Furlan, Phys. Lett. B 65, 163 (1976); Phys. Lett. B 72, 203 (1977); C. G. Callan, R. G. Dashen, and D. J. Gross, Phys. Lett. B 66, 375 (1977); Phys. Rev. D 17, 2717 (1978); 19, 1826 (1979); F. Lenz, J. W. Negele, and M. Thies, Phys. Rev. D 69, 074009 (2004); Nucl. Phys. B, Proc. Suppl. 140, 629 (2005).

[8] J. Hoek, M. Teper, and J. Waterhouse, Nucl. Phys. B288, 589 (1987); A. Hasenfratz, T. DeGrand, and D. Zhu, Nucl. Phys. B478, 349 (1996); P. de Forcrand, M. G. Perez, and 
I.-O. Stamatescu, Nucl. Phys. B, Proc. Suppl. 53, 557 (1997).

[9] M. N. Chernodub and M. I. Polikarpov, Proceedings of the NATO Advanced Study Institute on Confinement, Duality and Nonperturbative Aspects of QCD, Cambridge, England, 1997 (unpublished); hep-th/9710205; G. Bali, Proceedings of the 3rd International Conference on Quark Confinement and the Hadron Spectrum (Confinement III), Newport News, VA, 1998 (unpublished); hep-ph/9809351; R. Haymaker, Phys. Rep. 315, 153 (1999).

[10] See, for example, E.-M. Ilgenfritz, M.L. Laursen, G. Schierholz, M. Müller-Preussker, and H. Schiller, Nucl. Phys. B268, 693 (1986); M.-C. Chu, J. M. Grandy, S. Huang, and J. Negele, Phys. Rev. D 49, 6039 (1994); P. de Forcrand, M. G. Perez, and I.-O. Stamatescu, Nucl. Phys. B499, 409 (1997); D. A. Smith and M. J. Teper, Phys. Rev. D 58, 014505 (1998); A. Hasenfratz and C. Nieter, Nucl. Phys. B, Proc. Suppl. 73, 503 (1999); A. Ringwald and F. Schrempp, Phys. Lett. B 459, 249 (1999); E.-M. Ilgenfritz et al., Nucl. Phys. B, Proc. Suppl. 119, 754 (2003); C. Gattringer et al., Nucl. Phys. B, Proc. Suppl. 129, 653 (2004); F. Bruckmann et al.Nucl. Phys. B, Proc. Suppl. 140, 635 (2005).

[11] G. 't Hooft, in High Energy Physics, edited by A. Zichichi (Editrice Compositori, Bologna, 1976); S. Mandelstam, Phys. Rep. 23, 245 (1976); G. 't Hooft, Nucl. Phys. B190, 455 (1981); A. Kronfeld, G. Schierholz, and U. J. Wiese, Nucl. Phys. B293, 461 (1987); G. Ripka, Lecture Notes in Physics 639 (Springer, Berlin, 2004).

[12] Yu. Makeenko and A. A. Migdal, Nucl. Phys. B188, 269 (1981); A. A. Migdal, Phys. Rep. 102, 199 (1983); A. Dubin and Yu. Makeenko, in At The Frontier of Particle Physics, edited by M. Shifman (World Scientific, Singapore, 2002), Vol. 4, p. 2479.

[13] N. Christ and T. D. Lee, Phys. Rev. D 22, 939 (1980).

[14] R. Jackiw, in Proceedings of the 5th Jorge Andre Swieca Summer School, Campos do Jordao, Brazil, 1990, edited by O. J.P. Eboli, M. Gomes, and A. Santoro (World Scientific, Singapore, 1990), p. 731.

[15] H. Forkel (to be published).

[16] A. M. Polyakov, Phys. Lett. B 72, 477 (1978); L. Susskind, Phys. Rev. D 20, 2610 (1979); B. Svetitsky, Phys. Rep. 132, 1 (1986); M. Lüscher, R. Narayanan, P. Weisz, and U. Wolff, Nucl. Phys. B384, 168 (1992).

[17] D. I. Diakonov, Proceedings of the 4th St. Petersburg Winter School in Theoretical Physics, 1998; hep-th/ 9805137.

[18] D. Karabali, C. Kim, and V. P. Nair, Nucl. Phys. B524, 661 (1998); D. Karabali, C. Kim, and V. P. Nair, Phys. Lett. B 434, 103 (1998).

[19] H. Leutwyler, Nucl. Phys. B179, 129 (1981).

[20] See e.g. Proceedings of the International Workshop: Variational Calculations in Quantum Field Theory, Wangerooge, Germany, edited by L. Polley and D. E. L. Pottinger (World Scientific, Singapore, 1988); See also J. O. Akeyo, H. F. Jones, and C. S. Parker, Phys. Rev. D 51, 1298 (1995), for a corresponding lattice ansatz of the trial action.

[21] A. P. Szczepaniak, Phys. Rev. D 69, 074031 (2004).

[22] C. Feuchter and H. Reinhardt, Phys. Rev. D 70, 105021 (2004).
[23] D. Nolland, hep-th/0408075; I. I. Kogan and A. Kovner, Phys. Rev. D 51, 1948 (1995).

[24] I. I. Kogan and A. Kovner, Phys. Rev. D 52, 3719 (1995).

[25] For a discussion of computational problems with nonlinear sigma models and recent progress, see R. Ferrari, J. High Energy Phys. 08 (2005) 048.

[26] K. G. Wilson and J. B. Kogut, Phys. Rep. 12, 75 (1974); for a textbook exposition see M.E. Peskin and D. V. Schroeder, An Introduction to Quantum Field Theory (Addison-Wesley, Reading, MA, 1995).

[27] W. E. Brown and I. I. Kogan, Int. J. Mod. Phys. A 14, 799 (1999).

[28] M. Abramowitz and I. A. Stegun, Handbook of Mathematical Functions, National Bureau of Standards Applied Mathematics Series Vol. 55, (U.S. GPO, Washington, DC, 1972).

[29] I. Zahed and G. E. Brown, Phys. Rep. 142, 1 (1986).

[30] R. Hobart, Proc. R. Soc. London 82, 201 (1963); G. H. Derrick, J. Math. Phys. (N.Y.) 5, 1252 (1964).

[31] S. Coleman and E. Weinberg, Phys. Rev. D 7, 1888 (1973).

[32] N.S. Manton and P.M. Sutcliffe, Topological Solitons (Cambridge University Press, Cambridge, 2004).

[33] T. T. Wu and C. N. Yang, Phys. Rev. D 12, 3845 (1975).

[34] M.F. Atiyah, The Geometry and Physics of Knots (Cambridge University Press, Cambridge, 1990); L.H. Kauffman, Knots and Physics (World Scientific, Singapore, 1993).

[35] N. S. Manton and P. Ruback, Phys. Lett. B 181, 137 (1986).

[36] A. M. Polyakov, Nucl. Phys. B120, 429 (1977).

[37] L. D. Faddeev, Quantization of Solitons, Steklov Math. Institute and Institute for Advanced Study Report IAS-750570; in Proceedings of 18th International Conference on High Energy Physics, Tbilisi, USSR, 1976.

[38] L. D. Faddeev and A. J. Niemi, Nature (London) 387, 58 (1997); Phys. Rev. Lett. 82, 1624 (1999).

[39] B. Piette, B. Schroers, and W. Zakrzewski, Nucl. Phys. B439, 205 (1995).

[40] R. A. Battye and P. M. Sutcliffe, Phys. Rev. Lett. 81, 4798 (1998).

[41] A. F. Vakulenko and L. V. Kapitanskii, Dokl. Akad. Nauk SSSR 246, 840 (1979) [Sov. Phys. Dokl. 24, 433 (1979)]; A. Kundu and Yu.P. Rybakov, J. Phys. A 15, 269 (1982).

[42] R. S. Ward, Nonlinearity 12, 241 (1999).

[43] E. Langmann and A. Niemi, Phys. Lett. B 463, 252 (1999); A. J. Niemi, hep-th/0312133; L. D. Faddeev, A. J. Niemi, and U. Wiedner, Phys. Rev. D 70, 114033 (2004).

[44] H. Forkel, Phys. Rev. D 71, 054008 (2005).

[45] P. van Baal and A. Wipf, Phys. Lett. B 515, 181 (2001).

[46] V. N. Gribov, Nucl. Phys. B139, 1 (1978).

[47] A. A. Belavin, A. M. Polyakov, A. S. Schwarz, and Yu. S. Tyupkin, Phys. Lett B 59, 85 (1975).

[48] K. M. Bitar and S.-J. Chang, Phys. Rev. D 17, 486 (1978).

[49] W. E. Brown et al., Phys. Rev. D 59, 034015 (1999).

[50] C. G. Callan, R. G. Dashen, and D. J. Gross, Phys. Rev. D 17, 2717 (1978); 19, 1826 (1979).

[51] E. V. Shuryak, Phys. Rev. D 52, 5370 (1995); A.C. Aguilar, A. A. Natale, and P.S. Rodrigues da Silva, Phys. Rev. Lett. 90, 152001 (2003). 
[52] E. V. Shuryak and J. J. M. Verbaarschoot, Phys. Rev. D 52, 295 (1995).

[53] C. Michael and P.S. Spencer, Phys. Rev. D 52, 4691 (1995); T. DeGrand, A. Hasenfratz, and T. G. Kovacs, Nucl. Phys. B505, 417 (1997); Ph. de Forcrand, M. Garcia Perez, and I.-O. Stamatescu, Nucl. Phys. B499, 409 (1997); M. J. Teper, Phys. Rev. D 58, 014505
(1998).

[54] C. J. Houghton, N. S. Manton, and P. M. Sutcliffe, Nucl. Phys. B510, 507 (1998); J.-P. Longpré and L. Marleau, Phys. Rev. D 71, 095006 (2005), and references therein.

[55] T. Ioannidou, B. Piette, and W. J. Zakrzewski, J. Math. Phys. (N.Y.) 40, 6223 (1999). 